\title{
Villa of Theseus at Nea Paphos (Cyprus) Fourth-Early Fifth Century Numismatic Evidence for Architectural Transformations and Seismic Events
}

\author{
BARBARA LICHOCKA
}

\begin{abstract}
This paper presents coins unearthed in three separate places at the Villa of Theseus at Nea Paphos (Cyprus). With just a few exceptions they date to the fourth-early fifth centuries AD. Even though only some specimens are precisely identifiable, they deserve presentation since they may suggest termini post quem for the reconstructions and enlargement of the Villa of Theseus. At the same time, the numismatic evidence helps to support the hypothesis that more than one earthquake occurred in the late Roman period at Nea Paphos and caused the destruction of its residences in the whole or in part of the area.
\end{abstract}

Keywords: late Roman Cyprus, Nea Paphos, Villa of Theseus, North-western House, coins, earthquakes

Barbara Lichocka, Institute of Mediterranean and Oriental Cultures, Polish Academy of Sciences, Warsaw; blichocka@iksio.pan.pl; (D) 0000-0002-1437-9256

The largest residence discovered at Nea Paphos, and so far the largest residence on Cyprus as well, named the Villa of Theseus after the third century AD mosaic (in room 36), studied by Wiktor Andrzej Daszewski, ${ }^{1}$ was not originally created as such a vast complex (Fig. 1). Successive architectural alterations of the Villa were the subject of intensive research conducted by Stanisław Medeksza. ${ }^{2}$ This paper is dedicated to the memory of these two excellent scholars, whose research carried out over several years was crucial for the history of the Villa of Theseus.

Daszewski suggested four essential phases of its spatial development. ${ }^{3}$ He was of the opinion that the earlier part of the Villa of Theseus, the south wing, could possibly be dated to the third century AD. ${ }^{4}$ The enlarged edifice partially covered the ruins of earlier

1 Daszewski 1977; see also: Daszewski, Michaelides 1988: 54-57, Figs 23-24, end of the third or early fourth century AD.

2 A monographic study on Villa of Theseus in Nea Paphos was published in Polish; see: Medeksza 1992.

3 Daszewski 1968: 37.

4 Daszewski 1977: 12; Daszewski, Sztetyło 1988: 201. 


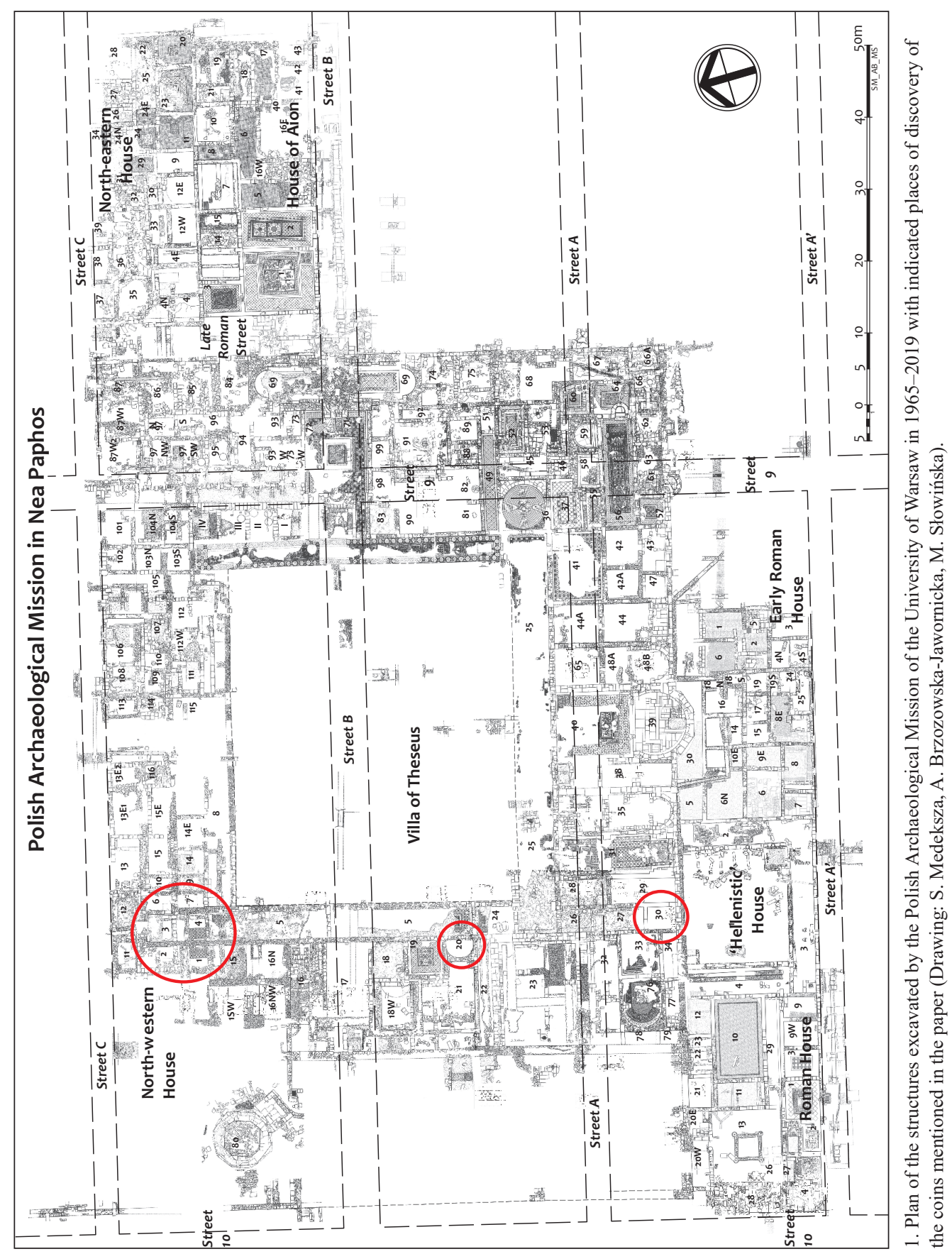


structures, including the building actually called the North-western House. ${ }^{5}$ Daszewski believed that the expansive Villa of Theseus could have been the residence of a highranking imperial officer. ${ }^{6}$

Medeksza supposed that the origin of the Villa of Theseus could be dated to the second half of the second century AD and was associated with a private house with a portico and two side extensions. Medeksza considered this building as phase I of the Villa. Phase II involved numerous renovations and structural changes realised in three stages, which resulted in a large peristyle residence combining private and official functions. ${ }^{7}$ Medeksza specified various renovations and remodellings which started already at the end of the third century and lasted until the third quarter of the fourth century AD. ${ }^{8}$ In the first stage of phase II, a peristyle was added to the north side of the second-century AD house, which became the south wing of the enlarged Villa. Extensive rebuilding covered certain parts of Hellenistic streets $(A, B, 9)$ and overlapped with a part of the North-western House (the so-called North villa), ${ }^{9}$ as well as further to the east with the neighbouring devastated structure on the north side, and the relicts of Hellenistic structures (I, II, III, IV) on the east side. Medeksza believed that some works had stopped by the fourth-century AD seismic events. ${ }^{10}$ It can also be assumed that after subsequent earthquakes several renovations were not only needed, but additionally created an opportunity for enlargement and remodelling of the residence.

When determining the dates of the earthquakes that had affected the Villa of Theseus, AD 332 and 342, Daszewski took numismatic evidence into account. ${ }^{11}$ However, the coin data should be examined more closely.

According to Henryk Meyza, only several undisturbed contexts in the Villa of Theseus, namely in rooms 4, 6, 7, 20 and 30, contained groups of coins which were highly likely collected at the original site of the loss. ${ }^{12} \mathrm{~A}$ few earlier specimens have also been found among the bronze and copper coins of low denominations typical of the fourth-early fifthcentury AD coinage in rooms 4, 6, 8 and 20.

The appearance of the coins minted throughout the fourth century AD and first years of the fifth century AD in the same archaeological contexts suggests that some of them remained in circulation over a relatively extended period of time. Coin finds from the North-eastern House, room 12E at Nea Paphos, ${ }^{13}$ as well as some from the West House, room 2 at Kourion ${ }^{14}$ seem to support this opinion.

5 Daszewski 1977: 12; Daszewski et al. 1984: 302-303; Daszewski, Sztetyłło 1988: 201-202, Figs 2-3.

${ }^{6}$ Daszewski 1972: 206.

7 Medeksza 1992: 48, 62, 64-65.

${ }^{8}$ Medeksza 1992: 35.

${ }^{9}$ Medeksza 1992: 31-32, 35-36; 1998: 28; for preliminary chronological sequence of the structures in the junction of the north and west wings of the Villa of Theseus drawn by P.M. Gartkiewicz, see: Młynarczyk 1990: 186, Fig. 23; Meyza 2007: 29, Fig. 4.

${ }^{10}$ Medeksza 1992: 32-33, 35, 40; 1998: 33-36.

${ }^{11}$ Daszewski 1977: 12; Medeksza 1992; 8, 35, indicates earthquakes, AD 332, 342 and AD 365-378.

${ }_{12}$ Meyza 2007: 26. According to Guest 2019: 244, coins are not often found in the place of loss.

${ }_{13}$ Lichocka, Meyza 2001: 189.

${ }^{14}$ Soren 1981: 126-130. 
Monetary reforms in the fourth century $\mathrm{AD}$ and the introduction of new types, often associated with the debasement of size and weight, generally resulted in the withdrawal of earlier coins. ${ }^{15}$ Some hoards illustrate that coins of Valentinian I largely displaced earlier currency. ${ }^{16}$ However, in the eastern Mediterranean the long-term circulation of fourth-century AD coins issued both under the Constantinian and Valentinian Dynasties was not unusual, even for a period of one hundred or more years, as is shown by finds from different sites in Israe ${ }^{17}$ or Egypt. ${ }^{18}$

In most cases, the time range of circulation of coin specimens remains unknown. It could have been from several years up to several decades. ${ }^{19}$ It seems impossible to set up a uniform model scheme. The appearance of a few specimens of earlier origin in the contexts predominated by late Roman imperial coins found in the Villa of Theseus deserves some consideration. ${ }^{20}$ Even withdrawn from use some time earlier, they may have survived and been kept by somebody, and eventually lost at the moment of construction, refurbishment or disaster. It cannot be ruled out that they had been lost or thrown away not in the place of discovery, but elsewhere, and moved during construction works on one of the residences, including levelling in preparation for the enlargement of the Villa of Theseus, perhaps after an earthquake. The appearance of earlier coins is not relevant with regard to the date of these events. Dating based on coins means establishing a date of issue, in the case of an assemblage of coins a date of issue of the latest specimen, and this date becomes a terminus post quem for an archaeological context.

As usual in the case of coins found in excavations, only some of the specimens unearthed in the Villa of Theseus are fully legible. A few specimens have survived with well-preserved mint-marks. Identification of a type or its variety, and consequently indication of the specific issue and its date, were not always possible. Traces of wear, independent of signs of corrosion processes, indicate that the coins were in use for quite a long period. Despite all well-known limitations, the study of the coins unearthed in, upon or below the floors of individual rooms of the Villa of Theseus plays a substantial role in dating the construction, renovation, and destruction of the rooms in which they were found.

\section{JUNCTION OF THE NORTH AND WEST WINGS (ROOMS 1, 1S, 3-4, 6-8)}

In the Villa of Theseus, famous for its mosaics, there were also very simple floors, which, as Daszewski observed, were made of 'clay and lime mixed together' (room 6), 'of beaten clay' (room 3), and possibly covered with 'terracotta slabs' (room 4). ${ }^{21}$ The modest floors

15 Burnett 1987: 132-133; cf. remarks in Guest 2019: 243-244, on money supply and fourth and early fifth-century AD reforms.

16 See: RIC VIII: 82.

17 Bijovsky 2000: 208.

18 Lichocka 2005: 764, 767, Table 1.

19 See, among others: Kraay 1956: 131; Sodini et al. 1980: 267; Augé 1987: 229.

${ }^{20}$ Cf. Walker 1997: 24-25.

21 Daszewski 1968: 40 and 41, respectively; Daszewski, distinguished eight types of floors in rooms 3, 5-10 (W.A. Daszewski, P. Gartkiewicz, Preliminary Report 1968, unpublished manuscript, unnumbered pages). 
of rooms 1S, 3 and 4 of the Villa of Theseus were superimposed on the floor paved with a geometric mosaic (partly preserved) belonging to the demolished early Roman Northwestern House. ${ }^{22}$ Only the layer of reddish earth above the bedrock was found under the floors of rooms 1, 2, 6 and probably of the northern part of room $5 .{ }^{23}$ The coins found in the layers between floor levels of the superimposed buildings, namely between the mosaic floor of the North-western House, whose complete plan is still unknown, ${ }^{24}$ and the remains of the floors in the north-western part of the Villa of Theseus, are essential for the dating of this part of the residence (phase II, stage 2).

The earliest late Roman coins known from the area of the north-western corner of the Villa of Theseus are the folles struck during the reign of Constantine I. The latest coin is one minted for Gratian, AD 367-375 (see Table 1). Both earlier and later fourth-century coins were unearthed on or below the floors ${ }^{25}$ of the Villa of Theseus (phase II). They all represent common types of the coinage circulating in these periods.

Three specimens of Securitas Reipublicae, Victory type, issued by Valentinian I and/or Valens between $\mathrm{AD} 364$ and $\mathrm{AD} 375$ were found in room 3 of the Villa of Theseus: two (nos 60/1966 and 61/1966) in a layer of greyish soil with mortar (floor or its substructure) above the early Roman mosaic, and one (no. 187/1971*.26 Fig. 2:14) in the lime mortar floor (washed out by rain?).

In room 4 two further specimens of Securitas Reipublicae type, AD 364-375, were discovered (nos 49/1966* and 50/1966; Fig. 2:10) in a layer (about 15-20cm thick) between the floor level of the Villa of Theseus (indicated by the partially preserved mosaics in room 5) and early Roman mosaics belonging to the North-western House. There were also two partly legible specimens struck under the Constantinian Dynasty (nos 47/1966 and 48/1966) and additionally a coin minted for Claudius II Gothicus (no. 52/1966). A Gloria Romanorum (no. 43/1966) type(?) with another, Constantinian Dynasty specimen, showing Spes Reipublice reverse type (no. 42/1966*; Fig. 2:6), were found about 1-2cm below the level of mosaics of the Villa of Theseus, under a layer of lime mortar.

The coins unearthed in brown soil covering the early Roman mosaic belonging to the North-western House, which was overlaid by room 4 of the Villa of Theseus come from the issues dated to the period ranging from $\mathrm{AD} 330-333$ to AD 367-375. Coins of Securitas Reipublicae type are the latest (nos 57/1966 and 59/1966*; Fig. 2:15). Two commemorative folles, one with Urbs Roma obverse type and a she-wolf suckling the twins on the reverse, dated to AD 330-333 (no. 55/1966*; Fig. 2:3), and the other, with Constantinopolis obverse type and Gloria Exercitus, Victoria on a prow reverse type dated to AD 332-333

${ }^{22}$ Daszewski 1968: 41, 50; Daszewski, Sztetyło 1988: 202; Medeksza 1992: 31-32; 1998: 28; Meyza 2007: 28; also: Mavrojannis 2016: 335. The mosaics unearthed in this structure were described as 'early Roman mosaic' in the Journal of Excavations and this term was used in Table 1.

23 Młynarczyk 1990: 185.

24 See the plan referred to in footnote 9, above.

25 Further designations of the floors were adopted after descriptions by Daszewski, the then director of excavations, made in field documentation, the Journal of Excavations.

26 The illustrated coins are indicated with an asterisk (*). 

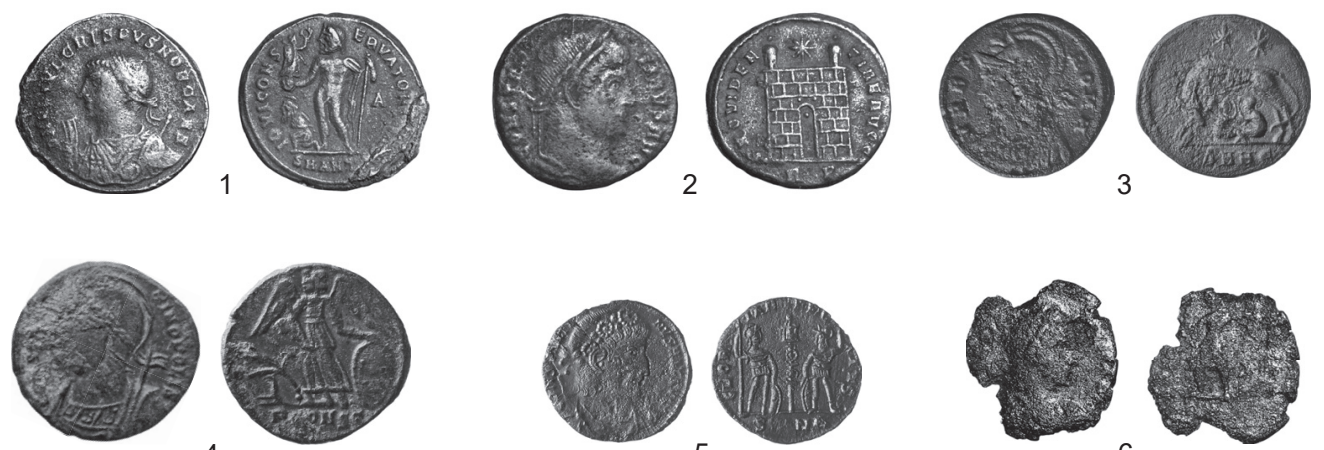

4
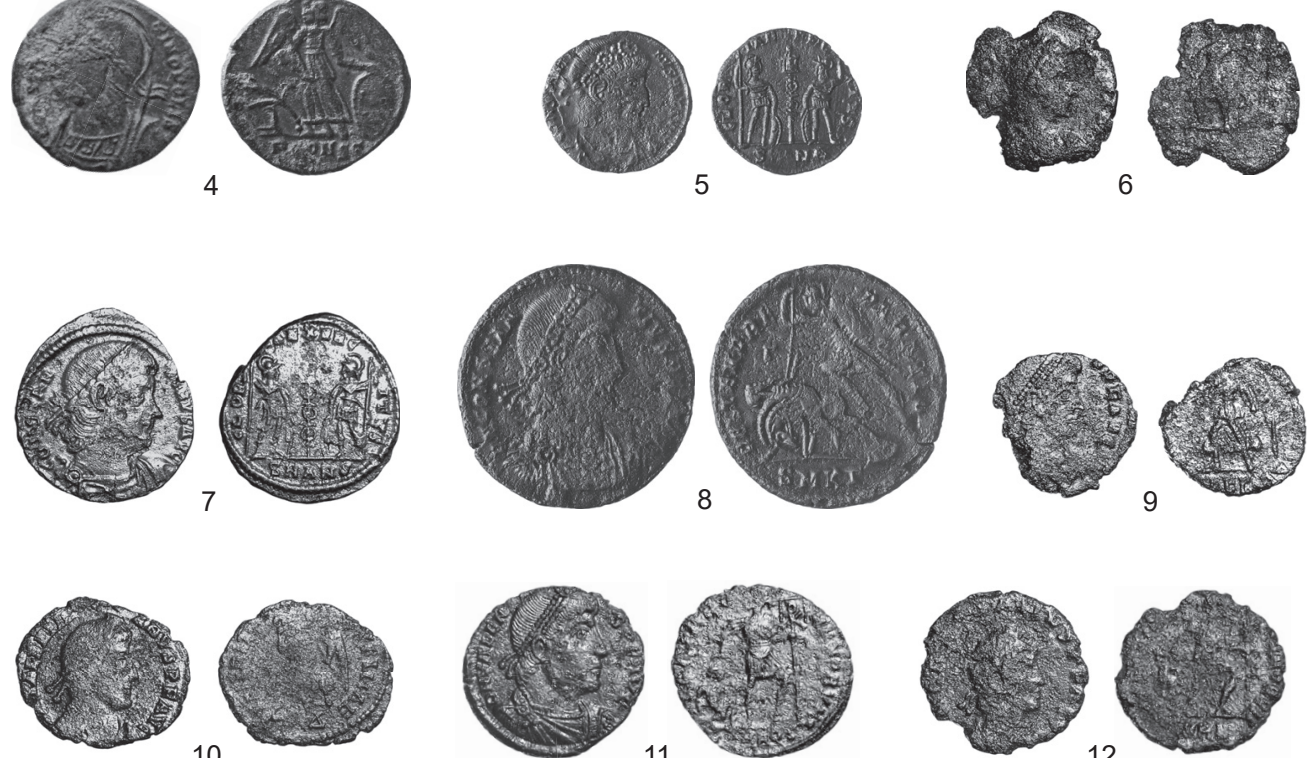

10
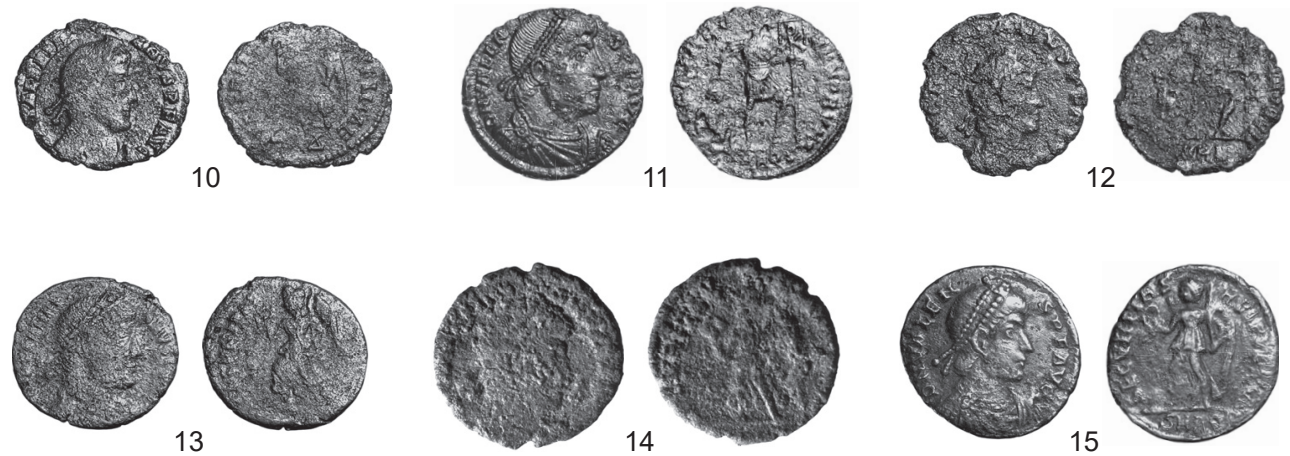

L $5 \mathrm{~cm}$

2. Coins found in the junction of the north and west wings of the Villa of Theseus, rooms 1, 1S, 3-4, 6-7: 1. no. 78/1966; 2. no. 72/1966; 3. no. 55/1966; 4. no. 56/1966; 5. no. 62/1966; 6. no. 42/1966; 7. no. 91/1966;

8. no. 63/1966; 9. no. 38/1966; 10. no. 49/1966; 11. no. 30/1966; 12. no. 41/1966; 13. no. 70/1966; 14. no. 187/1971; 15. no. 59/1966 (1-2, 6-7, 9-15. Phot. Z. Doliński; 3-5, 8. Phot. M. Jawornicki).

(no. 56/1966*; Fig. 2:4) are the earliest in this group. Apart from that, two specimens showing Fel Temp Reparatio, falling horseman type (nos 58/1966 and 63/1966*; Fig. 2:8) were unearthed in a layer of brown soil. Just above the early Roman mosaic there was one coin of Constantine I, showing Gloria Exercitus, the variety with one standard, AD 336-337 (no. 62/1966*; Fig. 2:5).

In room 1, the latest coin, AE4 of Valentinian I of Securitas Reipublicae type, dated to AD 364-375 (no. 70/1966*; Fig. 2:13), was discovered just under the stone pavement. 
It is not certain whether the place of discovery of Constantius II, Fel Temp Reparatio, the falling horseman type (no. 917/2007), that is, under the foundation of the north wall of the room was the original place of its loss or if it is an accidental fallout from the trench section.

A follis of Crispus, the Iovis Conservator type, dated to AD 317-320 (no. 78/1966*; Fig. 2:1) was unearthed in room $1 \mathrm{~S}$ below the floor level, above the level of the mosaic of the North-western House.

A coin of Constantine I, Providentia Augg, city-gate type, representing the issue dating to a time span from AD 324 to 330 (no. 72/1966*; Fig. 2:2) was discovered in room 7 adjacent to room 8 (north portico), at the level of the greyish pavement above the early Roman mosaic. Two specimens (nos 70a/1966 and 70b/1966) found in the south-western corner, in mortar with soil, just above the mosaic of the North-western House, date to AD 330-347. The specimen preserved on the wall with the stone door is Gloria Romanorum, an emperor in military dress dragging a captive type, issued for Valens, AD 364-378 (no. 30/1966*; Fig. 2:11).

Gloria Romanorum of Gratian (no. 41/1966*; Fig. 2:12) and Fel Temp Reparatio, the falling horseman type (no. 40/1966), were found in the south-eastern corner of room 6, at the floor level. Among the five coins retrieved from the extension of room 6, near the west wall on the upper greyish pavement, there were two coins of Gloria Exercitus, both varieties, with two standards (no. 85/1966) and with one standard (no. 87/1966), dated to AD 330-337. Apart from that, another coin belonging to the latter variety (no. 91/1966*; Fig. 2:7) was found separately.

The fourth-century AD coins from the north-western corner of the enlarged Villa of Theseus, especially from rooms 1, 3 and 4, appear to suggest that the North-western House was destroyed in the fourth century AD and in a relatively short period of time was covered by a new structure belonging to the Villa of Theseus. Among the coins unearthed in the fill under room 4, earlier than phase II, stage 2 of the Villa of Theseus, four coins dated to AD 364-375 (nos 49-50/1966, 57/1966, 59/1966) were discovered, so we may assume that the floor of room 4 may be dated to a period not earlier than the chronology of these specimens. Similar chronology is associated with the following coins: one found in room 1, under the pavement (no. 70/1966*; Fig. 2:13), three found in room 3 at the floor level (nos 60/1966, and 61/1966, 187/1971*; Fig. 2:14) and one discovered in room 6, at the floor level (no. 41/1966).

One late Ptolemaic bronze coin (no. 29/1966) was discovered in room 8 (north portico), at the level of the pavement. East of the wall with the door, just below the level of the mosaic of the Villa of Theseus, a follis Urbs Roma obverse type with Gloria Exercitus, two soldiers and one standard reverse type, dated to AD 336-337 (no. 39/1966*; Fig. 3:2) was discovered in greyish soil. Three coins: one of Sol Invictus type, minted for Constantine I, AD 316-317 (no. 67/1966*; Fig. 3:1) and two struck several decades later, a Gloria Exercitus, one standard variety, AD 346-348(?) (no. 66/1966*; Fig. 3:3), and Fel Temp Reparatio, falling horseman type (no. 68/1966) AD 348-361(?) were found c. $63 \mathrm{~cm}$ below the floor level, in brown soil reaching down to the bedrock. 


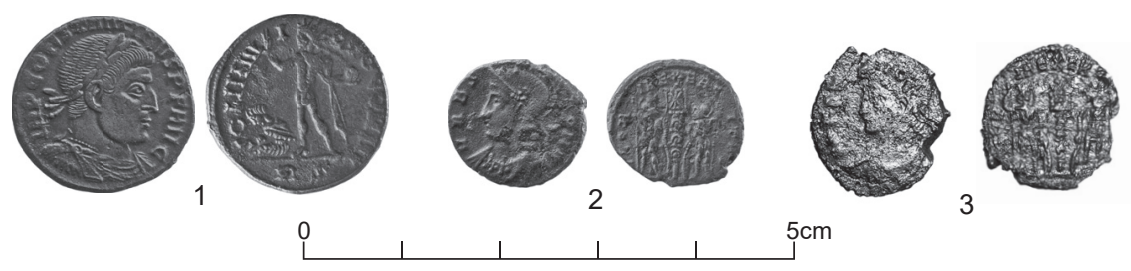

3. Coins found in the junction of the north and west wings of the Villa of Theseus, room 8: 1. no. 67/1966; 2 . no. 39/1966; 3. no. 66/1966 (1-2. Phot. M. Jawornicki; 3. Phot. Z. Doliński).

The coins found under the floor level of room 8 of the Villa of Theseus (nos 39/1966*, $66 / 1966 *, 68 / 1966$ the latest) undoubtedly belong to a period of some reconstructions or constructions added to the portico in the north, which may probably be dated not earlier than AD 352-361.

\section{WEST WING (ROOM 20)}

According to Medeksza, a relatively small apse room 20, adjacent to room 5 (west portico), which could be used as a lararium, was created (phase II, stage 2) after a series of earthquakes hit Paphos in the fourth century AD, up to the third quarter. ${ }^{27}$

Eleven coins were discovered in the rubble at the floor level (Table 2). Unfortunately, their poor state of preservation makes the identification of about half of the specimens uncertain or impossible. Leaving aside the sestertius of Philip the Arab (no. 86/1967), found in the course of cleaning the floor, AE4 Gloria Exercitus, variety with two standards between two soldiers, dated to AD 335-340 (no. 77/1967) is the earliest. Spes Reipublice, an emperor in military dress, holding a globe and spear type of Constantius II, dated to AD 355-361 (no. 79/1967*; Fig. 4) is the latest. There were also partly legible coins of Constantius II of Fel Temp Reparatio, the falling horseman type (nos 85/1967 and 87/1967).

One of AE4 Spes Reipublice type (no. 185/1971), was found under the stone blocking the entrance to the apse, which may suggest a terminus post quem of $\mathrm{AD} 355-361$ for the destruction, most likely from natural causes. ${ }^{28}$ This may point to speculation that the use of the apse may have ended in the second half of the fourth century, which in turn may raise the question of when the apse room was built. Was it really as late as Medeksza supposed? Single-coin dating, however, is risky. It is possible that the Spes Reipublice coin found in room 20 originated from the third quarter of the fourth century AD, but it remained a long time in circulation, and was lost only at the end of the fourth century or later, as may be suggested by the presence of the coin of probably Salus Reipublicae type, AD 383-395(?) (no. 76/1967), found in the rubble at the floor level. However, it cannot be ruled out that it was lost after the destruction of room 20.

\footnotetext{
${ }^{27}$ Medeksza 1992: 33, 40; 1998: 35.

${ }^{28}$ Unfortunately, there were no ceramic finds and we cannot confront our suppositions with pottery evidence.
} 


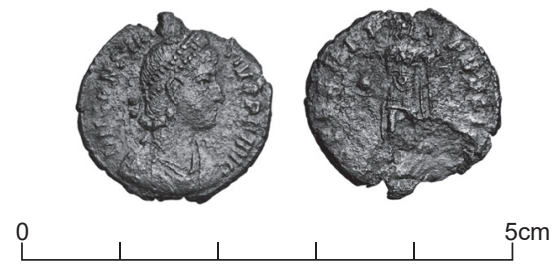

4. Coin found in the west wing of the Villa of Theseus, room 20: no. 79/1967 (Phot. Z. Doliński).

One of the heavily corroded coins (no. 82/1967) can be identified as 12 nummus piece of Heraclius (613-618), but not without a doubt. ${ }^{29}$ This coin, the only early seventh-century(?) specimen documented in room 20, might have been lost by a kind of visitor or squatter living in the residence, not necessarily in this wing. ${ }^{30}$

Summing up, it cannot be ruled out that the destruction of room 20 occurred shortly after the construction, and it could have happened at the end of the fourth or in the early fifth century AD.

\section{SOUTH WING (ROOM 30)}

Coins found in room 30 (Table 3) with the floor originally paved or made of beaten earth, ${ }^{31}$ range from the early fourth century to the early fifth century. Follis of Maximinus II Daza, Caesar, dated to AD 306-307 (no. 99/1968*; Fig. 5:1), found in the south-west corner below the floor level, is the earliest. A group of eight coins unearthed at the floor level, in daub, near rectangular blocks of stone comprise issues attributed to Constantius II (nos 125*-126/1969, 131/1969; Fig. 5:2), dated to AD 348-351, Valentinian II (nos 130/1969*; Fig. 5:3), dated to AD 383-388, and Arcadius or Honorius (nos 124/1969* and 128/1969; Fig. 5:4), dated to AD 395-401. One small module coin minted for Honorius (no. 181/1970) was found between the stones of the pavement. More coins were discovered at the level of the floor, the specimens being attributed to Arcadius, Honorius and/or Theodosius II (nos 133-134/1969, 139/1969), AD 395-408 and AD 406-408. They represent the following issues: Fel Temp Reparatio, an emperor with two captives, Gloria Romanorum, emperor with Victory on the ship, Virtus Exerciti, an emperor crowned by Victory. Perhaps one of these pieces (no. 142/1969) bears the Victory type of Salus Ripublicae, issued between AD 383 and 402. Most likely, these coins were not deposited intentionally, but accidentally were lost by one or more persons at the time of disaster.

29 Preliminary identification by Aleksandra Krzyżanowska, unpublished; Lichocka 2001: 256, no. 6.

${ }^{30}$ Daszewski 1970: 117, was of the opinion that the west wing of the Villa of Theseus was not occupied by squatters. According to Medeksza 1992: 45, squatters occupied the junction of the north and west wings of the Villa of Theseus already in the fifth century AD.

31 Daszewski 1970: 123. 

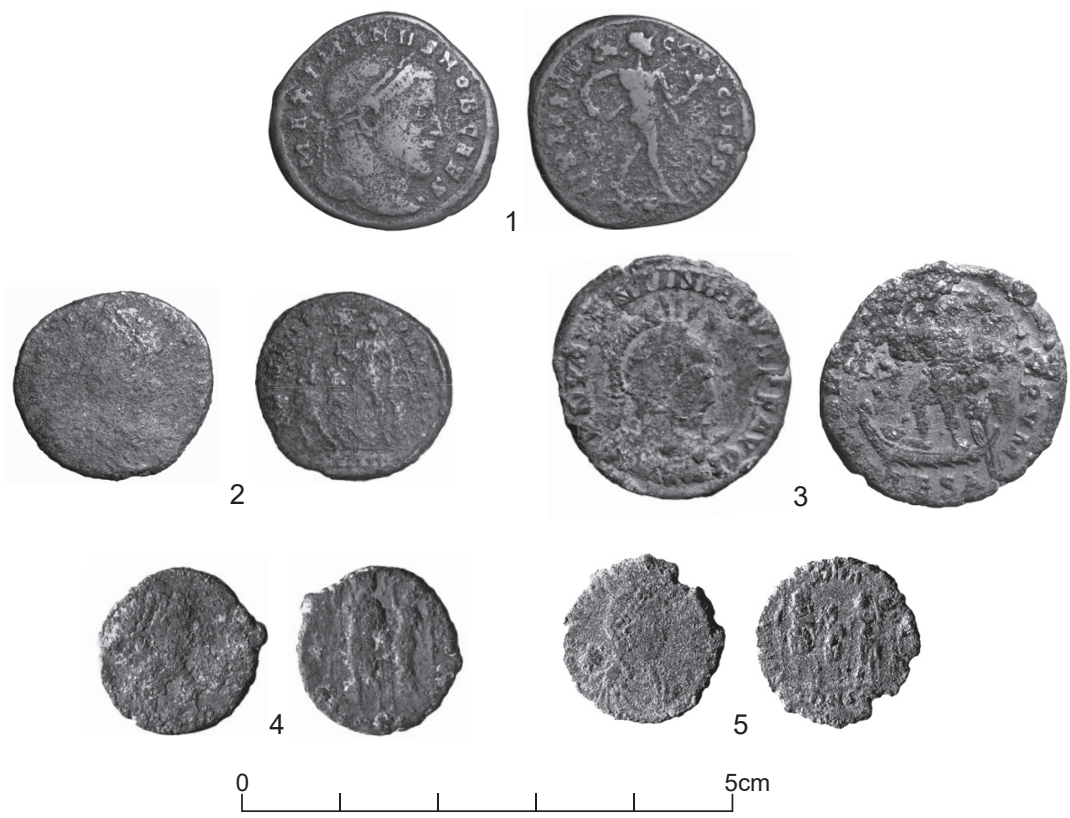

5. Coins found in the south wing of the Villa of Theseus, room 30: 1. no. 99/1968; 2. no. 125/1969; 3. no. 130/1969; 4. no. 124/1969; 5. no. 139/1969 (Phot. Z. Doliński).

Daszewski expected that room 30 had served as a storeroom. ${ }^{32}$ According to Medeksza, its construction or major reconstruction belongs to phase II, stage 1, and it took place most likely at the beginning of the fourth century $\mathrm{AD}^{33} \mathrm{~A}$ terminus post quem date for the destruction suggested by the coins is about a hundred years later, not before AD 406-408.

\section{CONCLUDING REMARKS}

Daszewski was of the opinion that the North-western House, could have been erected in the first century AD (although the mosaics were laid in the second century) ${ }^{34}$ and destroyed before the beginning of the third century AD. ${ }^{35} \mathrm{He}$ also supposed an early fourth century destruction. ${ }^{36}$ According to Medeksza, the North-western House was very likely destroyed at the beginning of the fourth century AD. ${ }^{37}$

Coins found below the floor level in the west part of room 8 appear to suggest the terminus post quem of $\mathrm{AD} 352-361$ for some construction works in the north portico of the

32 Daszewski 1970: 123.

33 Medeksza 1992: 35.

34 Daszewski 1968: 50; Daszewski, Sztetyło 1988: 202.

35 Daszewski 1977: 11.

36 Daszewski 1968: 50.

37 Medeksza 1992: 32. 
Villa of Theseus (phase II, stage 1?). Numismatic evidence from rooms 1, 2, 3, 6 indicates the terminus post quem of $\mathrm{AD} 364-375$ or $\mathrm{AD} 367-375$ for construction of some rooms in the junction of the north and west wings of the Villa of Theseus (phase II, stage 2), probably after an earthquake.

The suggestion that more than one disaster affected Nea Paphos seems to be indisputable. Most likely one of the earthquakes destroyed the North-western House. The termini post quem indicated by coins found in undisturbed strata in the House of Aion and North-eastern House are AD 355-361 and/or AD 364-367. ${ }^{38}$ A comparative study of pottery and coins found in both residences opens room for probable interpretations. One of the hypotheses is that the disaster took place around AD 380-400. ${ }^{39}$ These dates coincide with the termini post quem suggested by the coins discovered in the Villa of Theseus: AD 355-361 (room 20), AD 364-375 or AD 367-375 (rooms 1, 3-4), and AD 406-408 (room 30). It cannot be ruled out that some parts or even rooms of the Villa of Theseus were unequally affected by successive fourth-fifth century AD earthquakes of varying intensity and extent. ${ }^{40}$

\section{Acknowledgements}

I am very grateful to Aleksandra Brzozowska-Jawornicka for kindly providing me with the plan of the excavated structures at Nea Paphos (Maloutena) and adjusting it for this paper. All illustrations are courtesy of the Institute of Mediterranean and Oriental Cultures of the Polish Academy of Sciences and the Polish Centre for Mediterranean Archaeology of the University of Warsaw. My sincere thanks go to both institutions. I am indebted to Barbara Majchrzak for the proof-reading of the text.

\section{References}

Augé, Chr. 1987: La réutilisation des monnaies de bronze à l'époque impériale : quelques exemples proche-orientaux, [in:] Depeyrot, G., Hackens, T., Moucharte, G. (Eds), Rythmes de la production monétaire de l'Antiquité à nos jours. Actes du colloque international organisé à Paris du 10 au 12 janvier 1986, Louvain-la Neuve, 227-235

Bijovsky, G. 2000: The currency of the fifth century C.E. in Palestine - Some reflections in light of the numismatic evidence, INJ 14/2, 196-210

Burnett, A. 1987: Coinage in the Roman World, London

Daszewski, W.A. 1968: A preliminary report on the excavations of the Polish archaeological mission at Kato (Nea) Paphos in 1966 and 1967, RDAC 1968, 33-61

${ }^{38}$ Lichocka, Meyza 2001: 189.

${ }^{39}$ Lichocka, Meyza 2001: 207.

${ }^{40}$ This paper has been inspired by the article Lichocka, Meyza 2001, which in turn was inspired by a study presented in a joint presentation with Bounegru, F., Hanut, H., Meyza, H., Poblome, B. entitled 'Sherds and Coins - a Methodological Approach', at the $2^{\text {nd }}$ International Conference, Roman Crafts and Trade in the Roman East and West (ROCT), organised by Katholieke Universiteit Leuven, May 19-20, 2000. The author's part concerning coins, announced also by Lichocka 2005: 765, n. 17, finally was not submitted for print. 
Daszewski, W.A. 1970: Polish excavations at Kato (Nea) Paphos. Second Preliminary Report: seasons 1968 and 1969, RDAC 1970, 112-141

Daszewski, W.A. 1972: Polish excavations at Kato (Nea) Paphos in 1970 and 1971, RDAC 1972, 204-236

Daszewski, W.A. 1977: La mosaïque de Thésée, Nea Paphos II, Varsovie

Daszewski, W.A., Lichocka, B., Młynarczyk, J., Sztetyłło, Z. 1984: Fouilles polonaises à Kato Paphos. Chantier de Maloutena, RDAC 1984, 294-314

Daszewski, W.A., Michaelides, D. 1988: Mosaic floors in Cyprus, R. Farioli Campanati, R. (Ed.), Ravenna

Daszewski, W.A., Sztetyłło, Z. 1988: La région de Maloutena avant la construction de la Villa de Thésée, RDAC 1988/2, 195-203

Guest, P. 2019: The late Roman and early Byzantine coins, [in:] Poulter, A. (Ed.), The Transition to Late Antiquity on the Lower Danube: Excavations and survey at Dichin, a Late Roman to early Byzantine fort and a Roman aqueduct, Oxford, 237-279

Kraay, C.M. 1956: The Behaviour of Early Imperial Countermarks, [in:] Carson, R.A.G., Sutherland, C.H.V. (Eds), Essays in Roman Coinage Presented to Harold Mattingly, Oxford, 113-136

Lichocka, B. 2001: Le déclin de la Villa de Thésée à Nea Paphos. Les témoignages numismatiques, [in:] Papuci-Władyka, E., Śliwa, J. (Eds), Studia Archeologica Liber Amicorum Ianussio A. Ostrowski ab Amicis et Discipulus Oblatus / Prace dedykowane Profesorowi Januszowi A. Ostrowskiemu w Sześćdziesięciolecie urodzin, Kraków, 251-265

Lichocka, B. 2005: Late Roman coin-finds from Kom el-Dikka in Alexandria, [in:] Alfaro, C., Marcos, C., Otero, P. (Eds), XIII Congreso Internacional de Numismática, Madrid - 2003, Actas - Proceedings - Actes, in memoriam Carmen Alfaro Asins, Madrid, 763-769

Lichocka, B., Meyza, H. 2001: Seismic Events and the Evidence of Coins and Pottery. The Case of Destruction of the House of Aion in Paphos, EtudTrav XIX, 145-208 Mavrojannis, Th. 2016: La "Maison de Thésée" à Nea Paphos: le praetorium de 1'époque de Constantin, [in:] Balandier, C. (Ed.), Nea Paphos. Fondation et développement urbanistique d'une ville chypriote de l'Antiquité à nos jours. Études archéologiques, historiques et patrimoniales. Actes du $1^{\mathrm{er}}$ colloque international sur Paphos Avignon 30, 31 octobre et $1^{\text {er }}$ novembre 2012, Ausonius Mémoires 34, Bordeaux, 323-347

Medeksza, S. 1992: Willa Tezeusza w Nea Pafos. Rezydencja antyczna, Prace Naukowe Instytutu Historii Architektury, Sztuki i Techniki Politechniki Wrocławskiej 27 (Monografie 15), Wrocław

Medeksza, S. 1998: Willa Tezeusza w Nea Pafos na Cyprze: powstanie i fazy rozwoju, [in:] Daszewski, W.A., Meyza, H. (Eds), Cypr w badaniach polskich. Materiały z Sesji Naukowej zorganizowanej przez Centrum Archeologii Śródziemnomorskiej UW im. prof. K. Michałowskiego, Warszawa, 24-25 luty 1995, Warszawa, 25-50

Meyza, H. 2007: Cypriot Red Slip Ware. Studies on a Late Roman Levantine fine ware, Nea Paphos V, Varsovie 
Młynarczyk, J. 1990: Nea Paphos in the Hellenistic Period, Nea Paphos III, Varsovie Sodini, J.-P., Tate, G., Bavant, B., Bavant, S., Biscop, J.-L., Orssaud, D., Morrisson, C., Poplin, P. 1980: Déhès (Syrie du Nord). Campagnes I-III (1976-1978). Recherches sur l'habitat rural, Syria 57, 1-301

Soren, D. 1981: Earthquake: The Last Days of Kourion, [in:] Biers, J.C., Soren, D. (Eds), Studies in Cypriote Archaeology, Institute of Archaeology University of California, Monograph XVIII, Los Angeles, 117-133

Walker, A.S. 1997: Excavation coins: The use and misuse of numismatic evidence in archaeology, [in:] Sheedy, K.A., Papageorgiadou-Banis, Ch. (Eds), Numismatic Archaeology, Archaeological Numismatics. Proceedings of an International Conference Held to Honour Dr. Mando Oeconomides in Athens 1995, Oxbow Monographs 75, Oxford, 17-26 


\begin{tabular}{|c|c|c|c|c|c|c|c|c|}
\hline 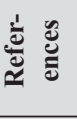 & & & 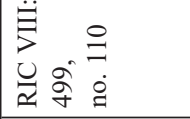 & & & 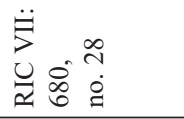 & & \\
\hline$\stackrel{\vec{E}}{\dot{E}}$ & & & 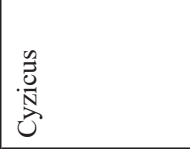 & & & 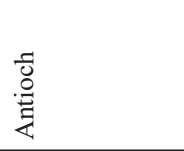 & & \\
\hline 节 हैّ & 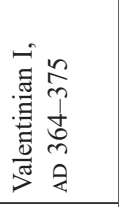 & 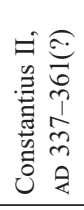 & 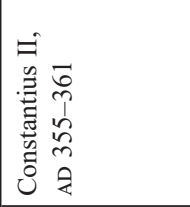 & & 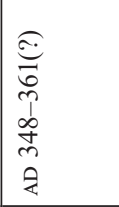 & 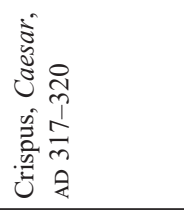 & 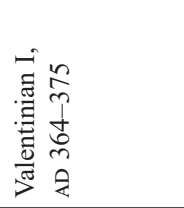 & 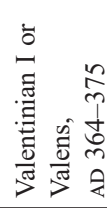 \\
\hline 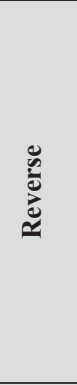 & 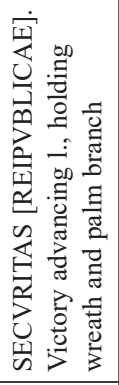 & $\begin{array}{l}\frac{0}{0} \\
\frac{0}{00} \\
\stackrel{0}{\equiv}\end{array}$ & 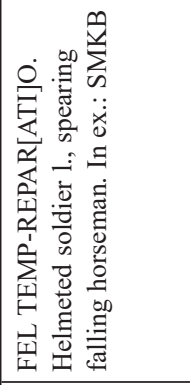 & $\begin{array}{l}\frac{0}{0} \\
\frac{0}{00} \\
\stackrel{0}{\Xi} \\
\end{array}$ & 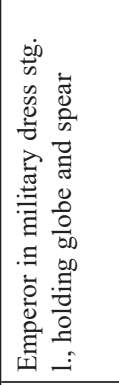 & 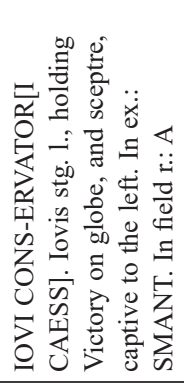 & 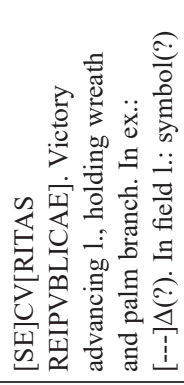 & 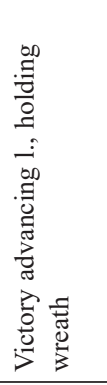 \\
\hline 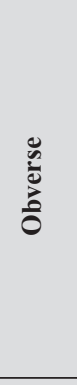 & 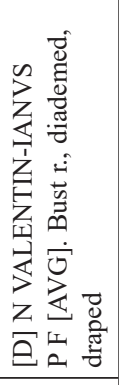 & 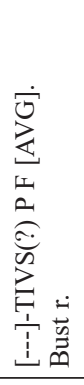 & 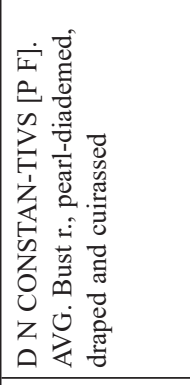 & $\begin{array}{l}\frac{0}{0} \\
\frac{0}{0.0} \\
\stackrel{\equiv}{\equiv}\end{array}$ & 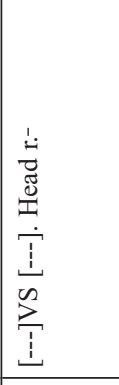 & 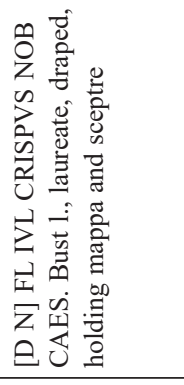 & 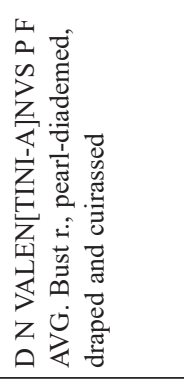 & 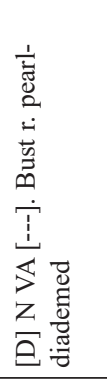 \\
\hline 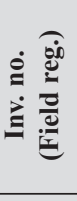 & 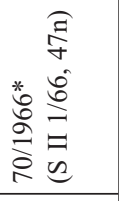 & 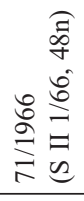 & 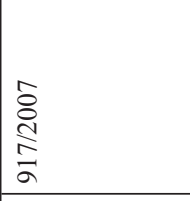 & $\begin{array}{l}\hat{8} \\
\stackrel{1}{\infty} \\
\frac{\infty}{a}\end{array}$ & $\begin{array}{l}\hat{\sigma} \\
\stackrel{N}{1} \\
\infty \\
\infty \\
\sigma\end{array}$ & 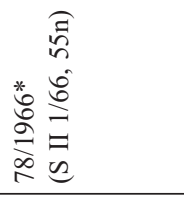 & 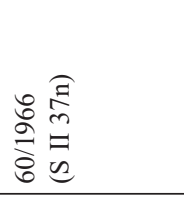 & 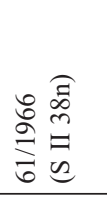 \\
\hline 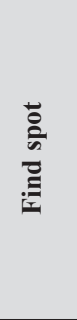 & 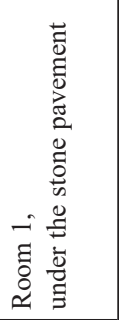 & 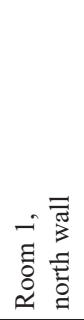 & 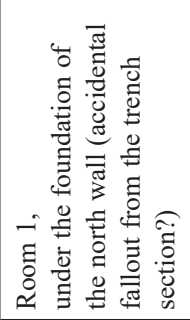 & 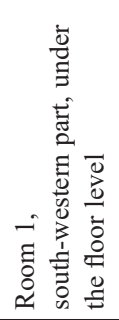 & 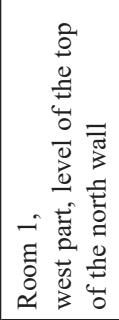 & 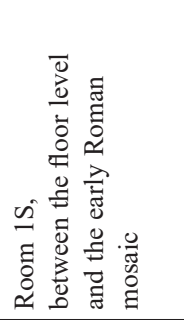 & 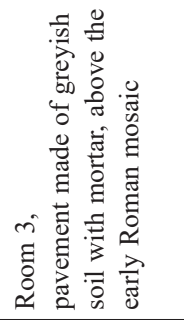 & $\begin{array}{ll}\text { mi } & 0 \\
\Xi & 0 \\
0 & 0 \\
0 & \pi \\
\simeq & \pi\end{array}$ \\
\hline
\end{tabular}




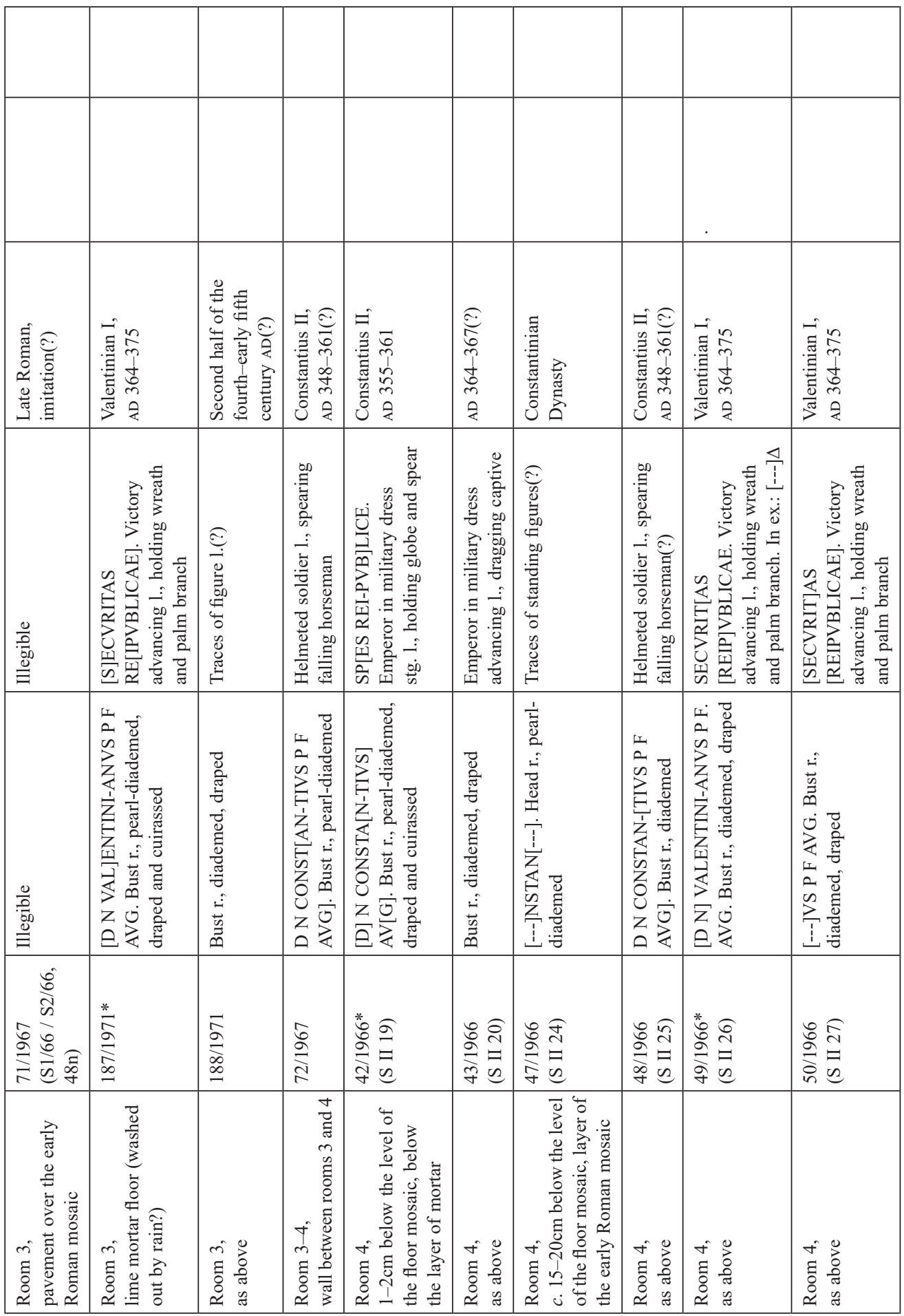




\begin{tabular}{|c|c|c|c|c|c|c|c|c|c|}
\hline 竞 & & 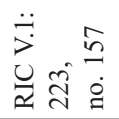 & & 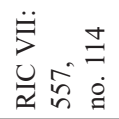 & 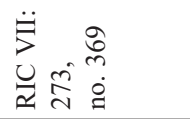 & & & 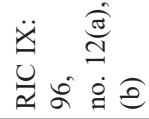 & 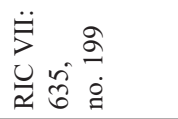 \\
\hline$\vec{\Xi}$ & & 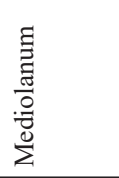 & & 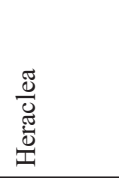 & $\frac{e}{3}$ & & & $\frac{. \frac{\pi}{0}}{\frac{3}{3}}$ & 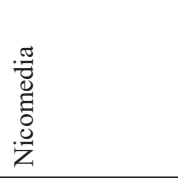 \\
\hline 这 气 & 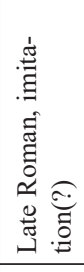 & 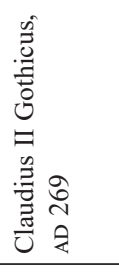 & & 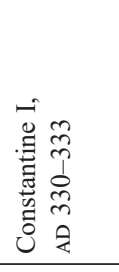 & 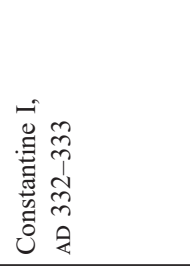 & 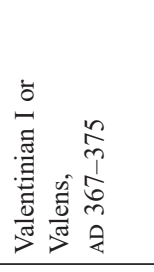 & 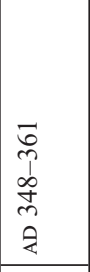 & 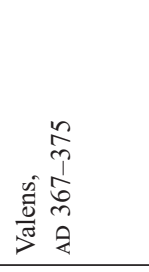 & 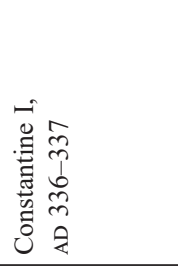 \\
\hline 苋 & 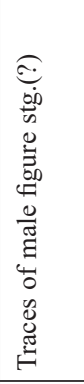 & 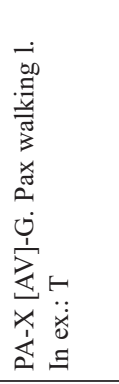 & $\begin{array}{l}\stackrel{0}{0} \\
\text { क0.0 } \\
\stackrel{0}{=}\end{array}$ & 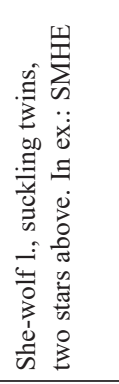 & 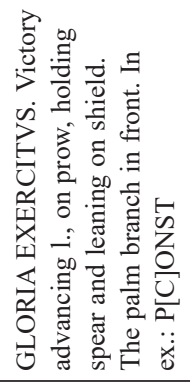 & 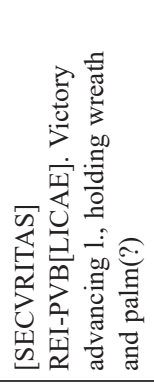 & 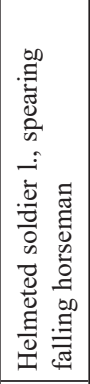 & 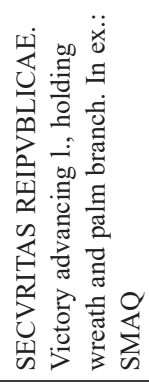 & 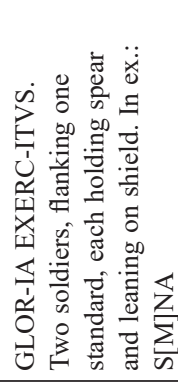 \\
\hline 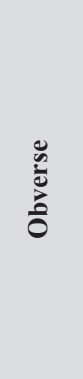 & 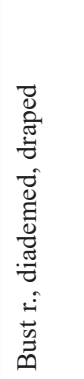 & 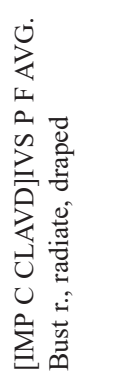 & $\begin{array}{l}\stackrel{0}{\overrightarrow{0}} \\
\stackrel{0}{0.0} \\
\stackrel{\Xi}{\equiv}\end{array}$ & 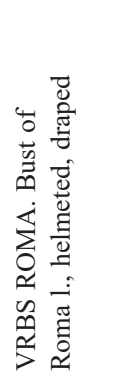 & 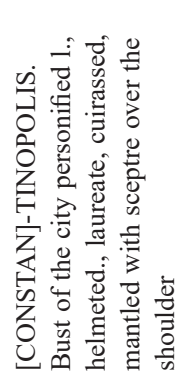 & 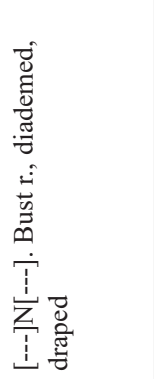 & 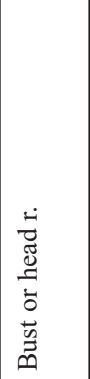 & 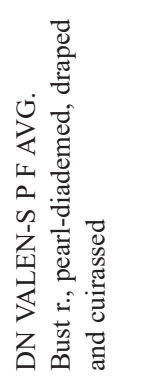 & 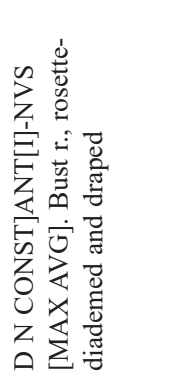 \\
\hline 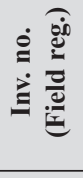 & 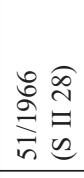 & 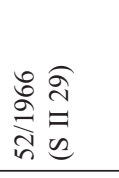 & 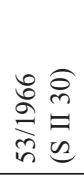 & 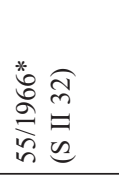 & 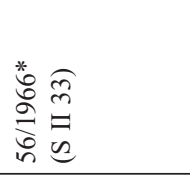 & 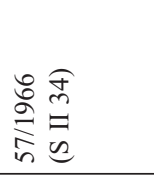 & $\begin{array}{ll}0 & 0 \\
0 & n \\
\circ & = \\
\infty & 0 \\
n & 0\end{array}$ & 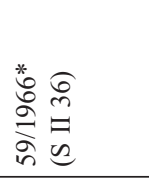 & 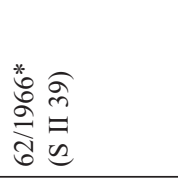 \\
\hline 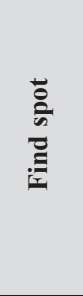 & 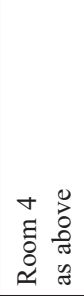 & 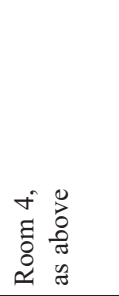 & 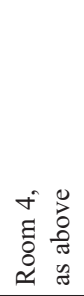 & 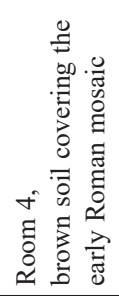 & 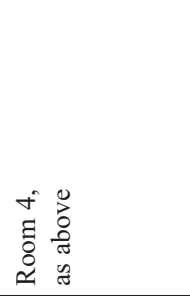 & 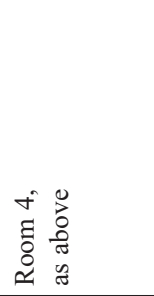 & 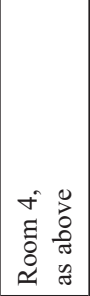 & 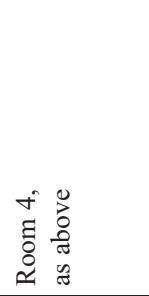 & 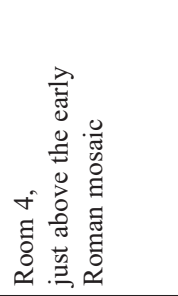 \\
\hline
\end{tabular}




\begin{tabular}{|c|c|c|c|c|c|c|c|c|c|}
\hline 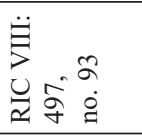 & & & & 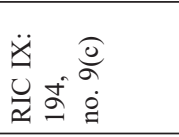 & & 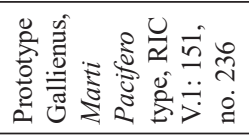 & & 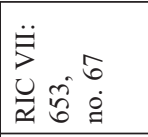 & \\
\hline 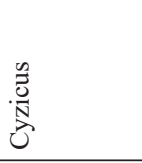 & & & & 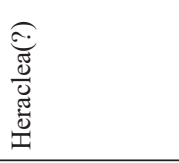 & & & & 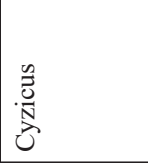 & \\
\hline 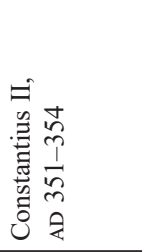 & 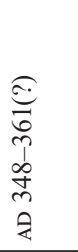 & 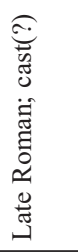 & 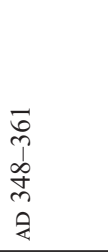 & 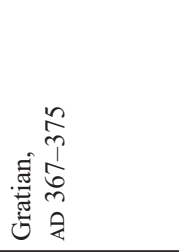 & 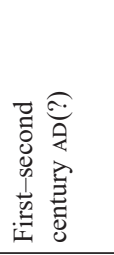 & 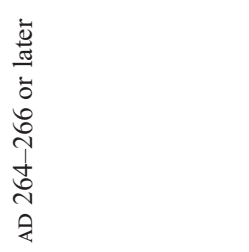 & 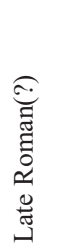 & 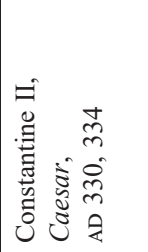 & 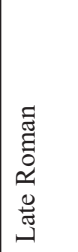 \\
\hline 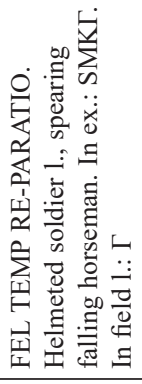 & 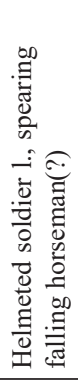 & 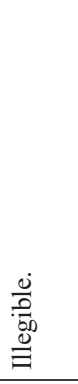 & 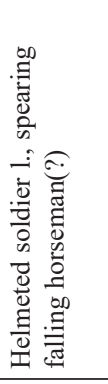 & 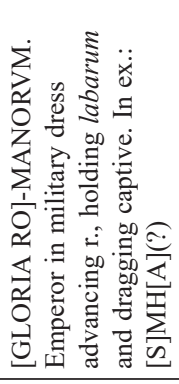 & 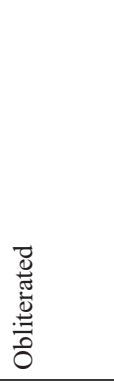 & 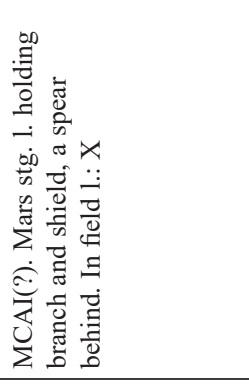 & $\begin{array}{l}\frac{0}{0} \\
\frac{0}{00} \\
\stackrel{0}{=}\end{array}$ & 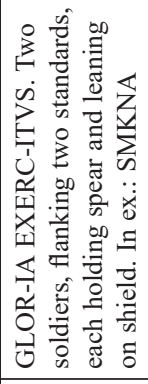 & \begin{tabular}{|l}
$\stackrel{0}{\circ}$ \\
$\frac{0}{0.0}$ \\
$\stackrel{0}{\equiv}$ \\
\end{tabular} \\
\hline 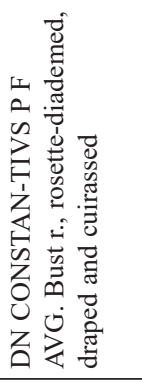 & 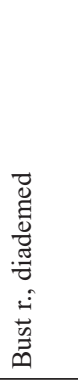 & 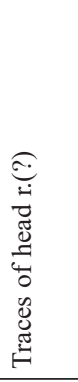 & 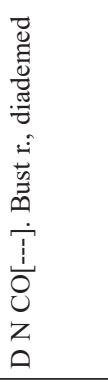 & 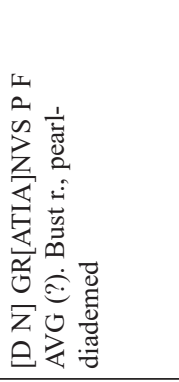 & 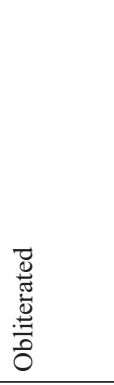 & 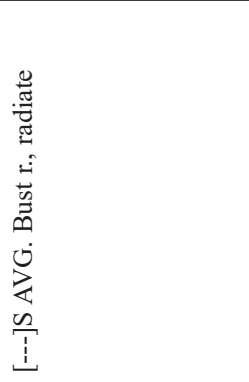 & $\begin{array}{l}\frac{0}{0} \\
\frac{0}{00} \\
\stackrel{0}{\equiv}\end{array}$ & 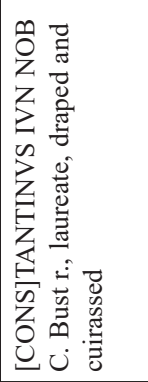 & 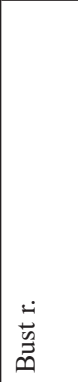 \\
\hline 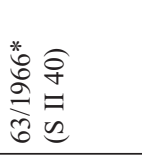 & 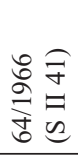 & 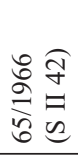 & 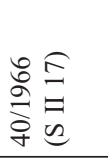 & 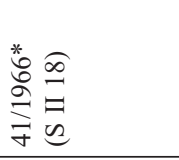 & 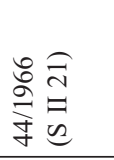 & 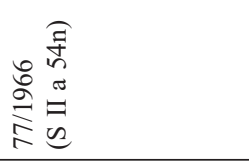 & 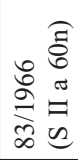 & 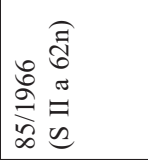 & 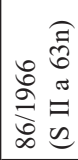 \\
\hline 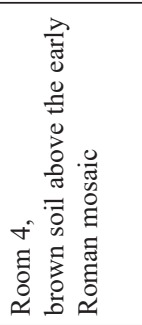 & 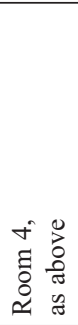 & 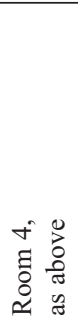 & 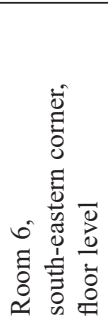 & 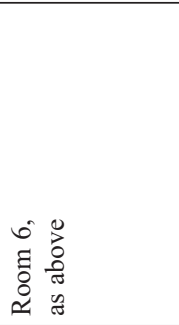 & 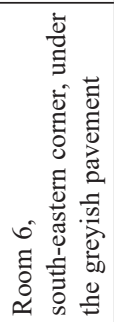 & 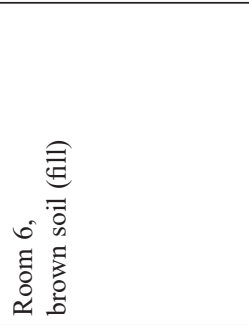 & 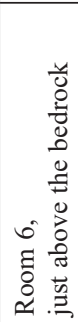 & 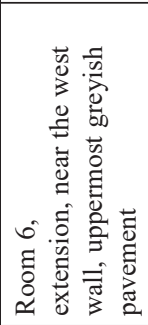 & 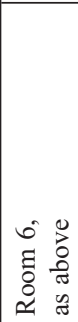 \\
\hline
\end{tabular}




\begin{tabular}{|c|c|c|c|c|c|c|c|}
\hline 离 & 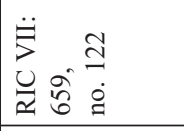 & & & 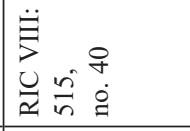 & 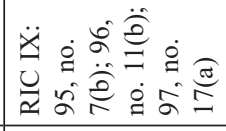 & 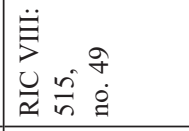 & 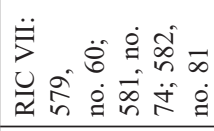 \\
\hline$\stackrel{\vec{E}}{\underline{\Sigma}}$ & 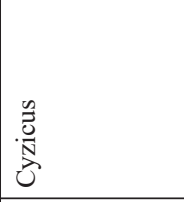 & & & 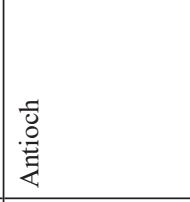 & $\frac{\frac{\pi}{2}}{\frac{0}{3}}$ & 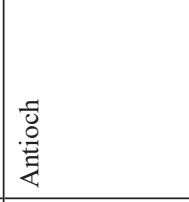 & 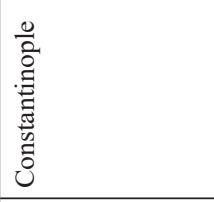 \\
\hline 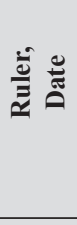 & 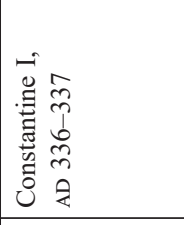 & 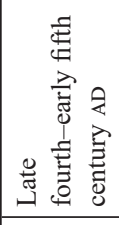 & 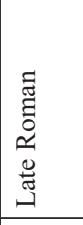 & 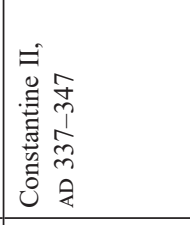 & 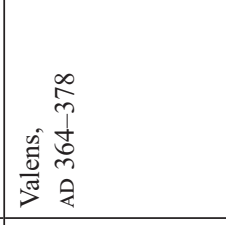 & 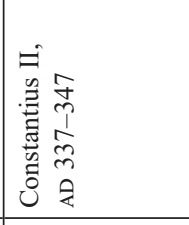 & 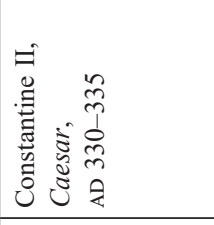 \\
\hline 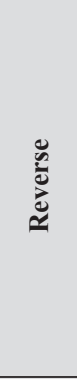 & 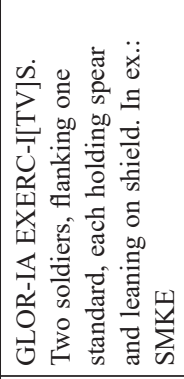 & 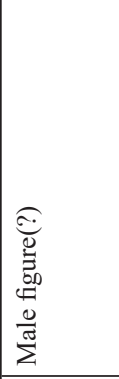 & $\begin{array}{l}\frac{0}{0} \\
\frac{0}{00} \\
\stackrel{0}{=}\end{array}$ & 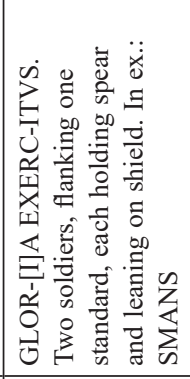 & 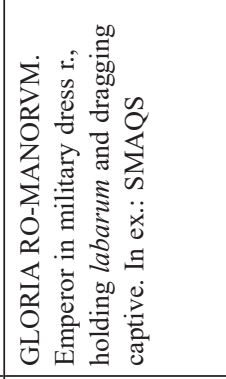 & 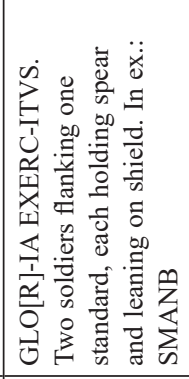 & 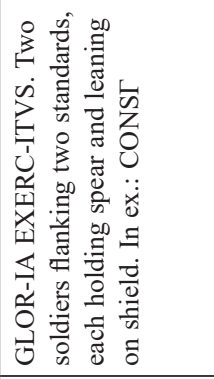 \\
\hline $\begin{array}{l}: \\
\frac{0}{0} \\
\frac{0}{0} \\
0\end{array}$ & 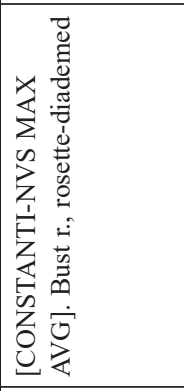 & 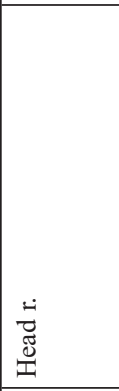 & 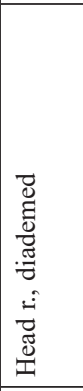 & 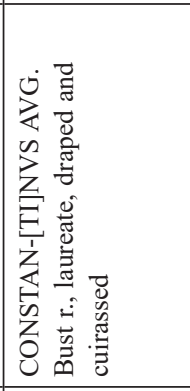 & 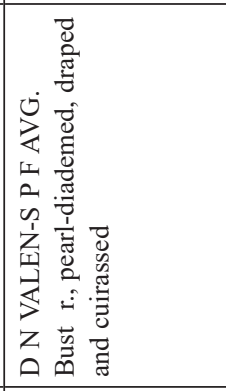 & 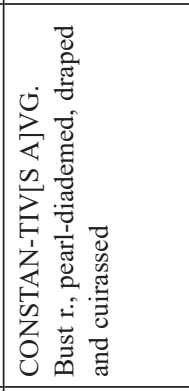 & 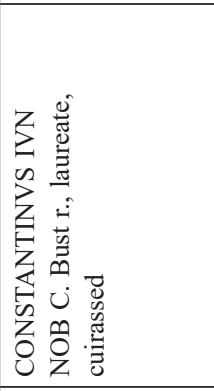 \\
\hline 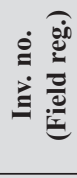 & 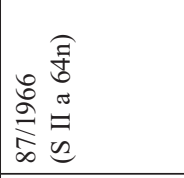 & $\begin{array}{ll} & \widehat{\sigma} \\
0 & 0 \\
0 & 0 \\
\infty & = \\
\infty & 0 \\
\infty & 0\end{array}$ & 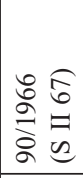 & 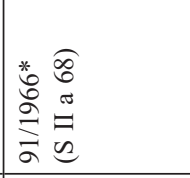 & 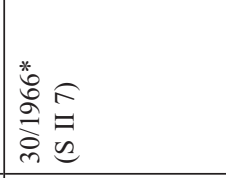 & 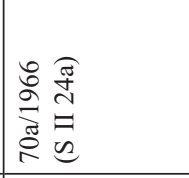 & 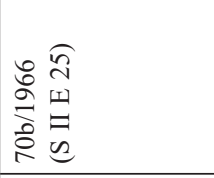 \\
\hline 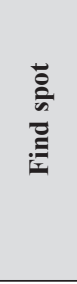 & 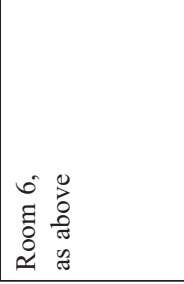 & 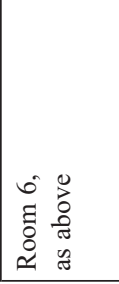 & $\begin{array}{ll}6 & 0 \\
\Xi & 0 \\
0 & 0 \\
0 & 0 \\
\simeq & 0 \\
\end{array}$ & 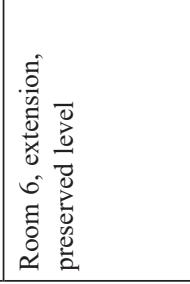 & 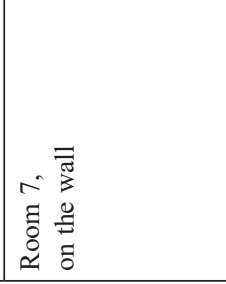 & 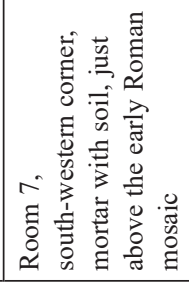 & 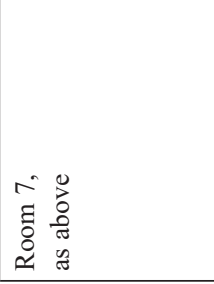 \\
\hline
\end{tabular}




\begin{tabular}{|c|c|c|c|c|c|c|c|}
\hline 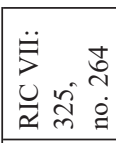 & & & & 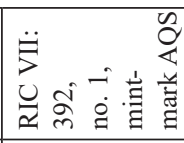 & & & \\
\hline ڤ્ & 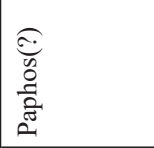 & & & $\frac{.0}{0}$ & & & \\
\hline 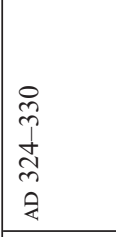 & 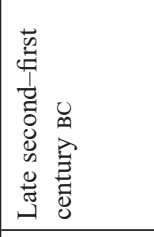 & 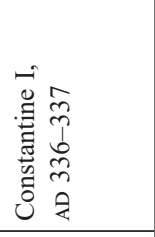 & 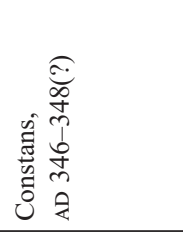 & 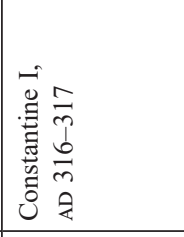 & 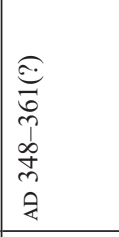 & & $\begin{array}{l}5 \\
0 \\
0 \\
0 \\
0 \\
5 \\
5 \\
0 \\
0\end{array}$ \\
\hline 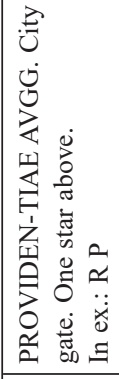 & 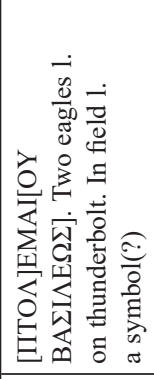 & 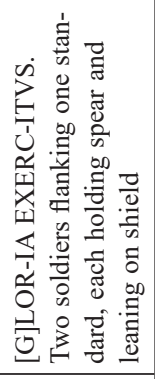 & 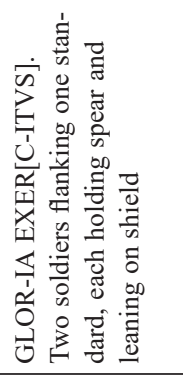 & 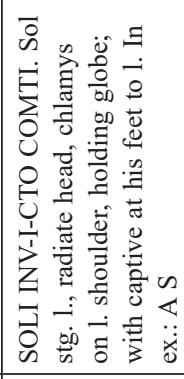 & 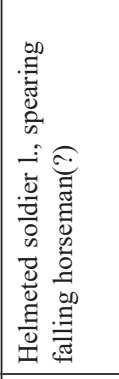 & $\begin{array}{l}\frac{0}{0} \\
\frac{0}{0} \\
\stackrel{0}{=}\end{array}$ & 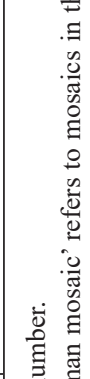 \\
\hline 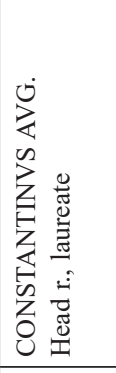 & 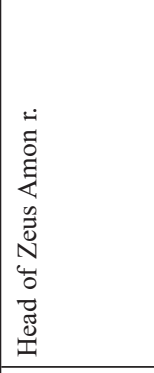 & 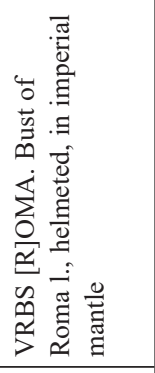 & 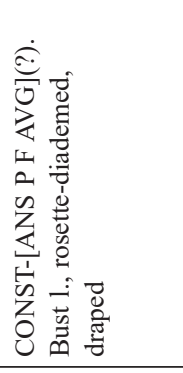 & 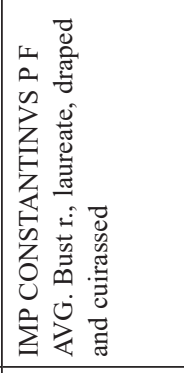 & 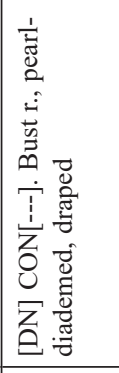 & 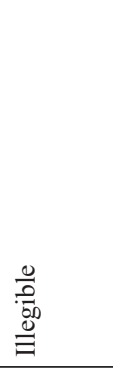 & 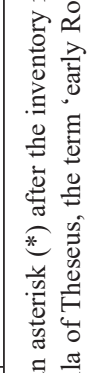 \\
\hline 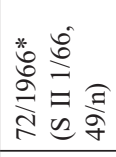 & 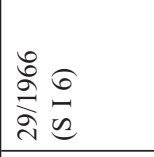 & 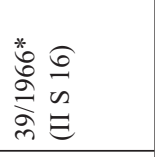 & 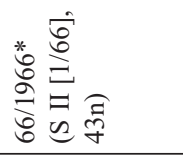 & 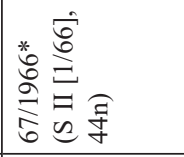 & 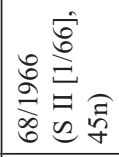 & 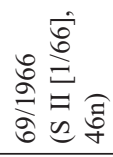 & 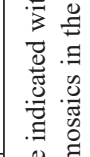 \\
\hline 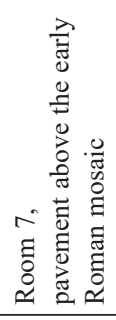 & 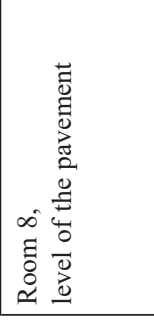 & 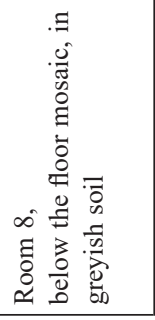 & 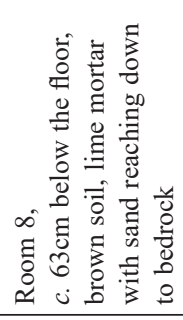 & 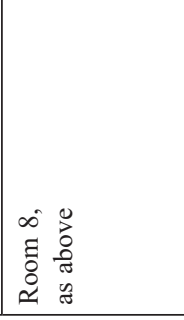 & 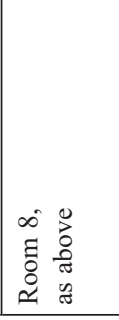 & 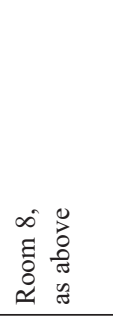 & 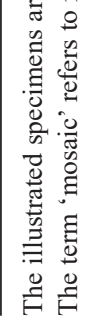 \\
\hline
\end{tabular}




\begin{tabular}{|c|c|c|c|c|c|c|c|c|c|c|}
\hline 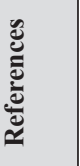 & & & & & & & & & & 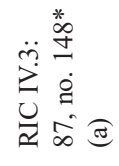 \\
\hline$\stackrel{\vec{E}}{\dot{E}}$ & & 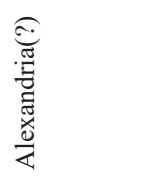 & & & & 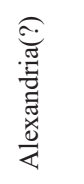 & & & & ஜ̆ \\
\hline 节 & 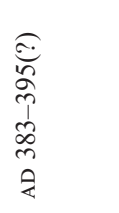 & 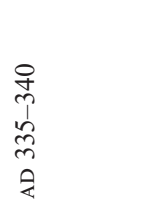 & & 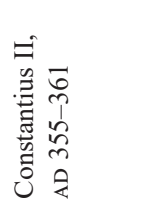 & 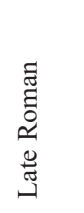 & 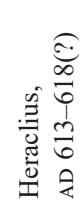 & & 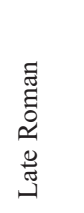 & $\begin{array}{l}\overrightarrow{0} \\
\infty \\
\infty \\
\infty \\
m \\
\&\end{array}$ & 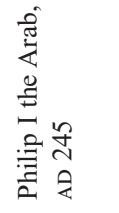 \\
\hline 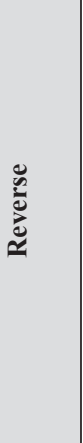 & 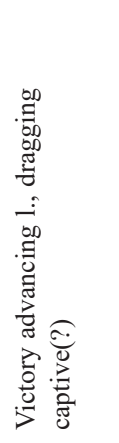 & 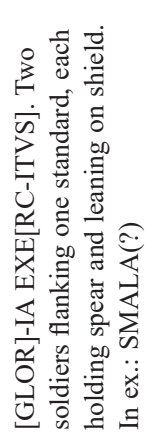 & $\begin{array}{l}\frac{0}{0} \\
\frac{0}{00} \\
\stackrel{\Xi}{=}\end{array}$ & 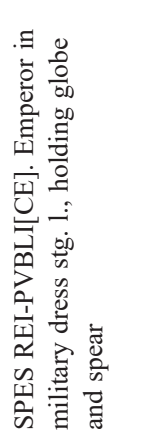 & $\begin{array}{l}\frac{0}{0} \\
\stackrel{0}{0} \\
\stackrel{0}{\equiv}\end{array}$ & 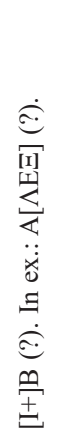 & $\begin{array}{l}\frac{0}{0} \\
\stackrel{0}{0} \\
\stackrel{0}{=}\end{array}$ & 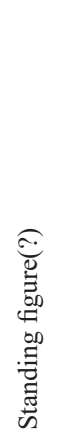 & 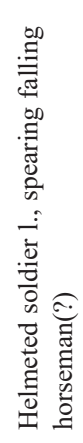 & 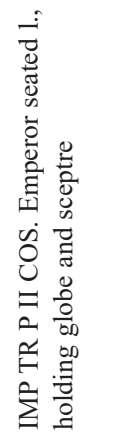 \\
\hline $\begin{array}{l}\frac{0}{2} \\
\frac{2}{0} \\
0 \\
0\end{array}$ & $\begin{array}{l}\frac{0}{0} \\
\text { क्D. } \\
\stackrel{0}{=}\end{array}$ & $\begin{array}{l}\text { : } \\
\stackrel{\Xi}{0} \\
\stackrel{0}{5}\end{array}$ & $\begin{array}{l}\frac{0}{0} \\
\frac{0}{00} \\
\stackrel{\Xi}{\equiv}\end{array}$ & 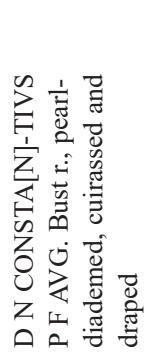 & $\begin{array}{l}\frac{0}{0} \\
\stackrel{0}{0} \\
\stackrel{0}{=}\end{array}$ & $\begin{array}{l}\frac{0}{0} \\
\stackrel{0}{0} \\
\stackrel{0}{=}\end{array}$ & $\dot{\ddot{J}}$ & 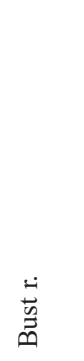 & 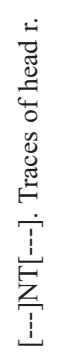 & 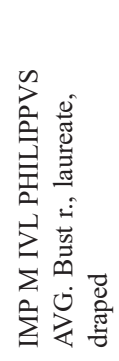 \\
\hline 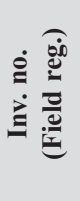 & $\frac{\hat{\&}}{2}$ & 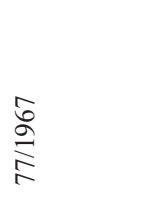 & $\underset{\infty}{\stackrel{0}{\infty}}$ & 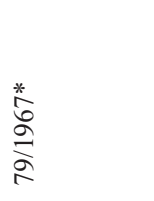 & $\begin{array}{l}\hat{0} \\
\stackrel{\infty}{\infty}\end{array}$ & 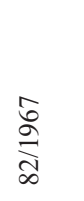 & 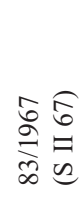 & $\frac{\hat{\&}}{\stackrel{\infty}{\infty}}$ & $\frac{\hat{\sigma}}{\stackrel{\infty}{\infty}}$ & $\frac{\hat{\&}}{\stackrel{\infty}{\infty}}$ \\
\hline $\begin{array}{l}\overrightarrow{0} \\
\vdots \\
\vdots \\
\vdots \\
\vdots\end{array}$ & 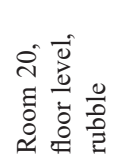 & 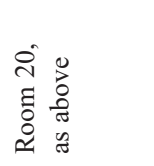 & 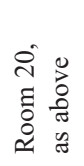 & 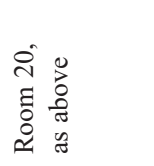 & 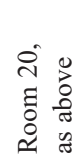 & 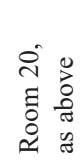 & 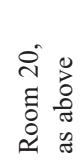 & 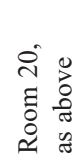 & 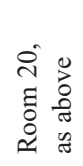 & 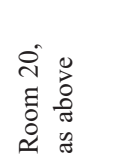 \\
\hline
\end{tabular}




\begin{tabular}{|c|c|}
\hline & 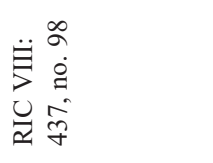 \\
\hline & 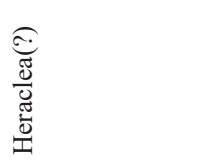 \\
\hline 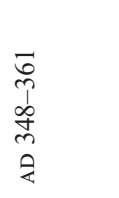 & 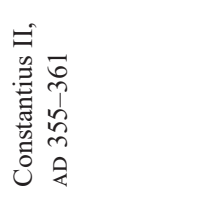 \\
\hline 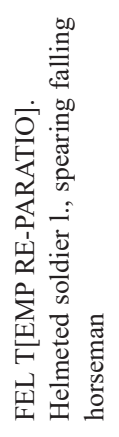 & 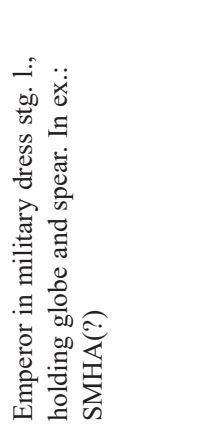 \\
\hline 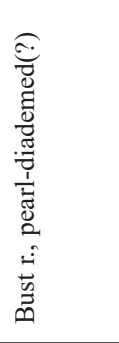 & 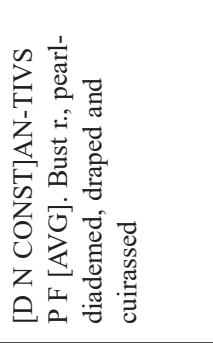 \\
\hline$\underset{\infty}{\stackrel{\infty}{\infty}}$ & 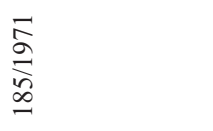 \\
\hline 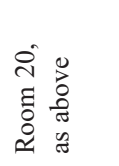 & 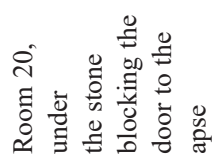 \\
\hline
\end{tabular}




\begin{tabular}{|c|c|c|c|c|c|}
\hline 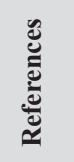 & 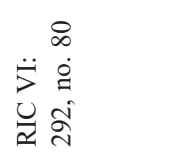 & & 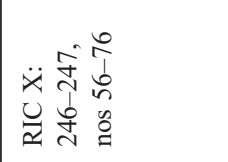 & 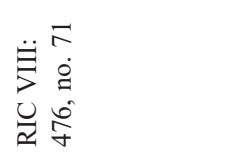 & 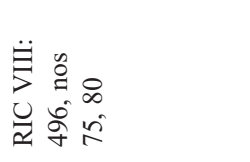 \\
\hline $\overrightarrow{\underline{z}}$ & $\widehat{\overparen{\mathscr{G}}}$ & & & 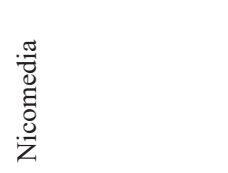 & 离 \\
\hline 苞 & 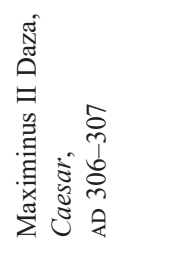 & 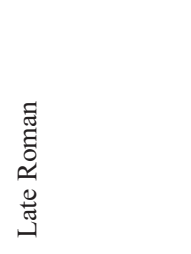 & 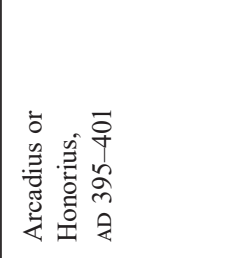 & 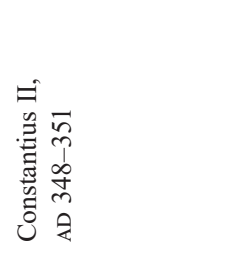 & 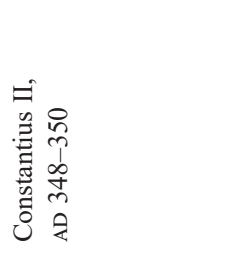 \\
\hline 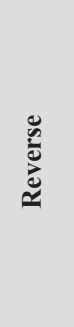 & 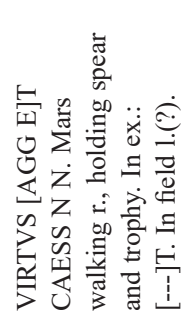 & $\begin{array}{l}\stackrel{\dot{j}}{\mathrm{D}} \\
\stackrel{\text { gn }}{=}\end{array}$ & 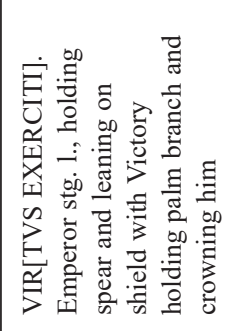 & 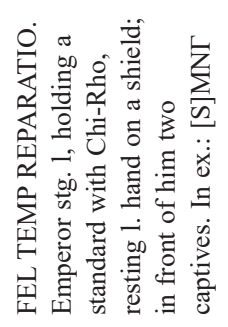 & 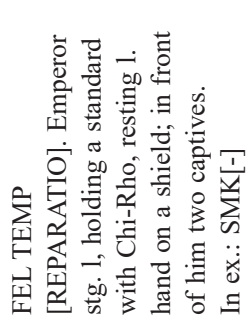 \\
\hline 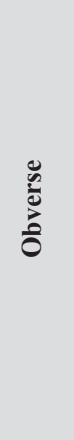 & 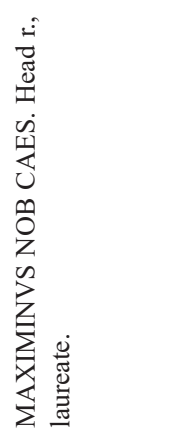 & 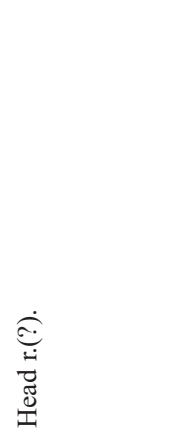 & 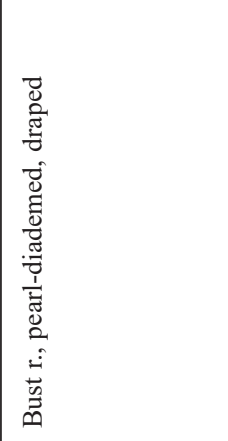 & 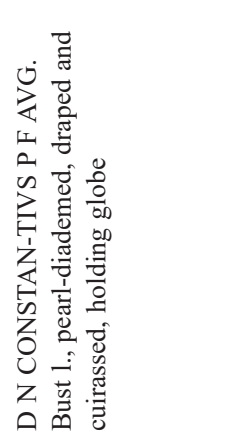 & 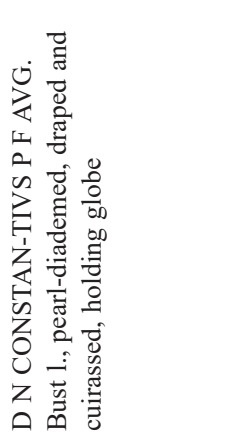 \\
\hline $\begin{array}{l}\dot{\Xi} \\
\dot{\Xi}\end{array}$ & $\begin{array}{l}\text { *o } \\
\text { oे } \\
\stackrel{2}{\alpha}\end{array}$ & 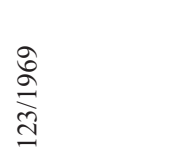 & 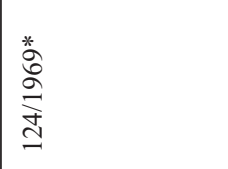 & 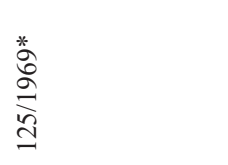 & 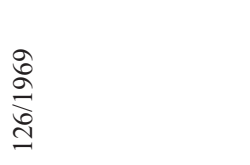 \\
\hline 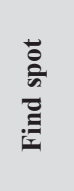 & 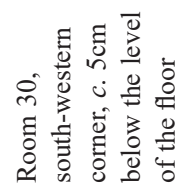 & 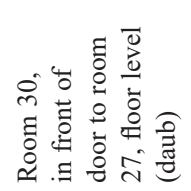 & 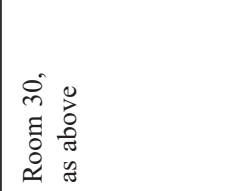 & 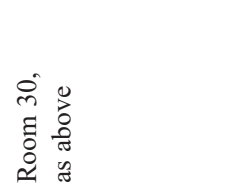 & 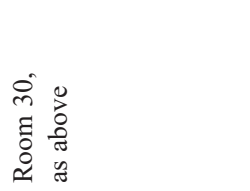 \\
\hline
\end{tabular}




\begin{tabular}{|c|c|c|c|c|c|c|}
\hline & 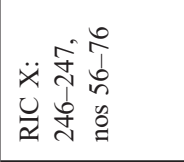 & 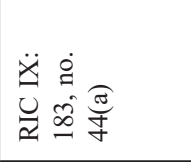 & 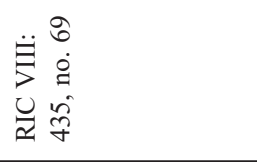 & 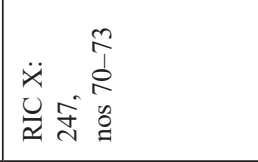 & 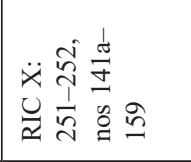 & \\
\hline & & 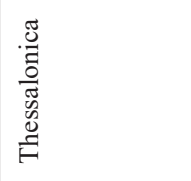 & 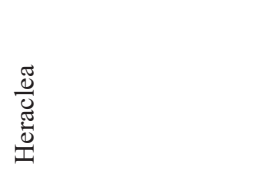 & $\begin{array}{l}\bar{y} \\
\text { 章 } \\
\end{array}$ & & \\
\hline & 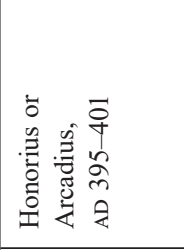 & 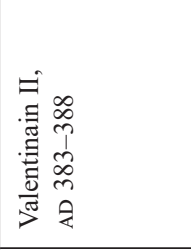 & 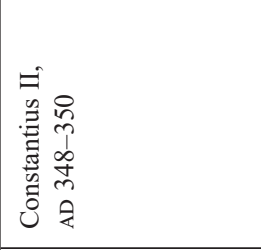 & 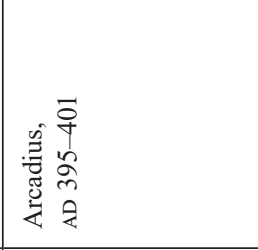 & 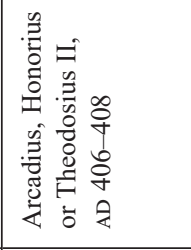 & 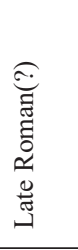 \\
\hline $\begin{array}{l}\frac{0}{\circ} \\
\stackrel{0}{\circ} \\
\stackrel{e}{=}\end{array}$ & 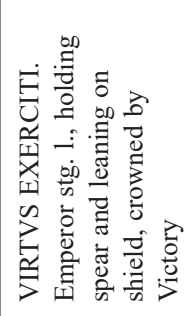 & 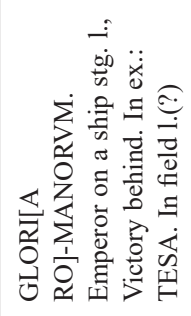 & 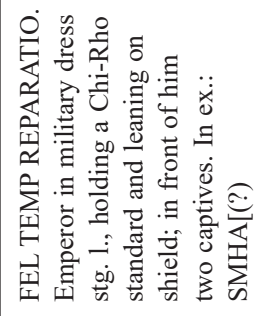 & 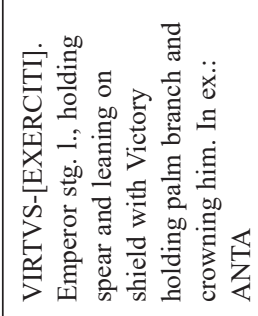 & 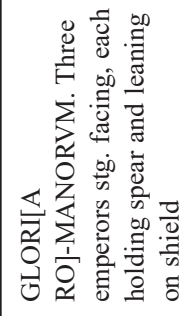 & $\begin{array}{l}\frac{0}{0} \\
\frac{00}{0} \\
\stackrel{\equiv}{=}\end{array}$ \\
\hline 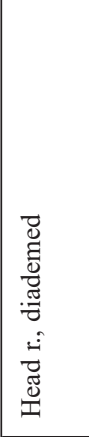 & 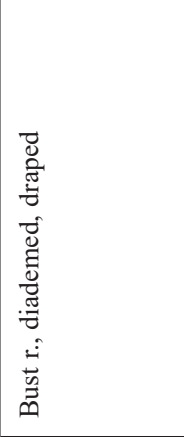 & 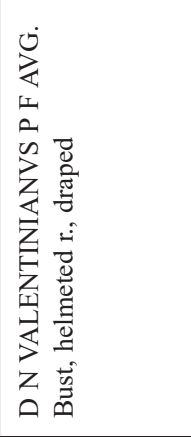 & 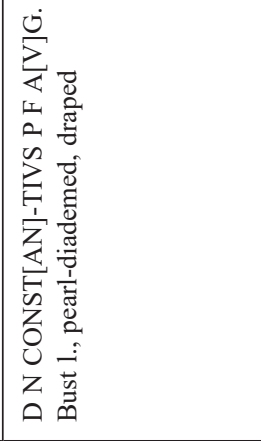 & 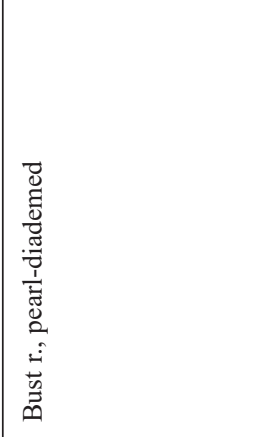 & $\begin{array}{l}\frac{0}{0} \\
\frac{0}{0.0} \\
\stackrel{\equiv}{=}\end{array}$ & 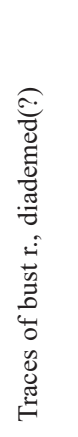 \\
\hline 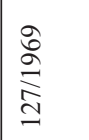 & $\begin{array}{l}\stackrel{8}{\circ} \\
\stackrel{2}{\infty} \\
\text { d }\end{array}$ & 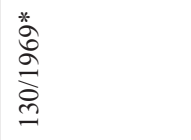 & 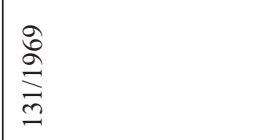 & $\frac{\stackrel{\partial}{\circ}}{\stackrel{ल}{m}}$ & 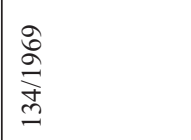 & 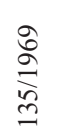 \\
\hline 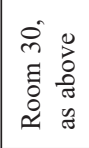 & 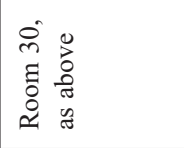 & 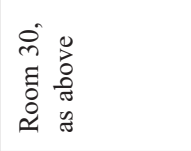 & 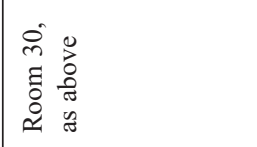 & 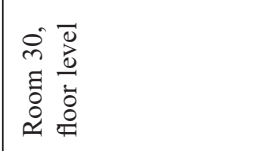 & 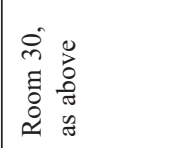 & 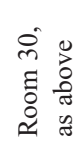 \\
\hline
\end{tabular}




\begin{tabular}{|c|c|c|c|c|c|c|}
\hline 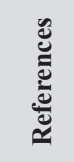 & & & 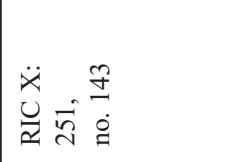 & & & 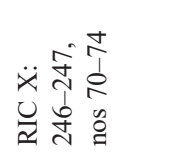 \\
\hline $\overrightarrow{\mathrm{E}}$ & & & 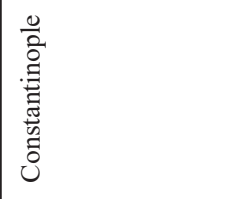 & & & 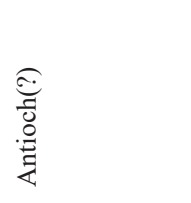 \\
\hline 这 & 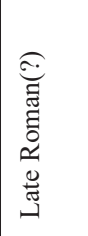 & 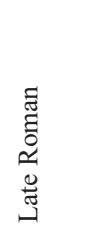 & 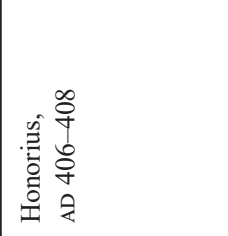 & 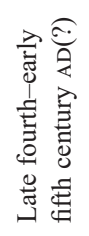 & 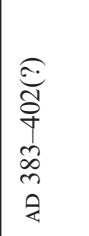 & 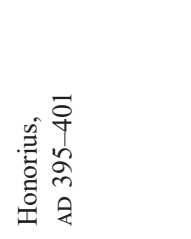 \\
\hline 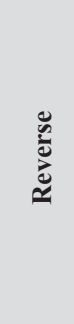 & $\begin{array}{l}\frac{0}{0} \\
\text { 总 } \\
\stackrel{\Xi}{=}\end{array}$ & 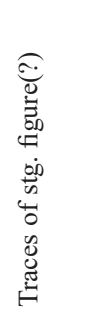 & 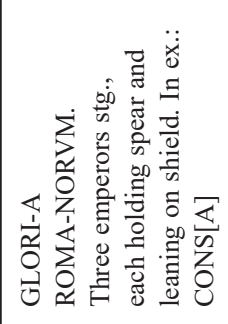 & 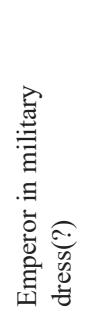 & 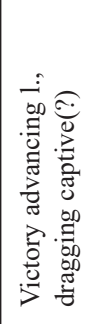 & 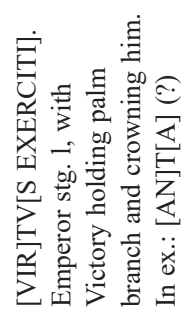 \\
\hline 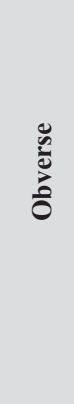 & 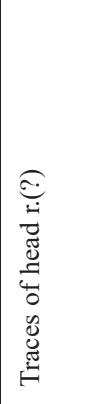 & 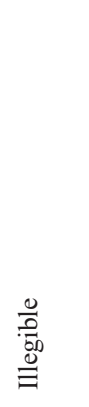 & 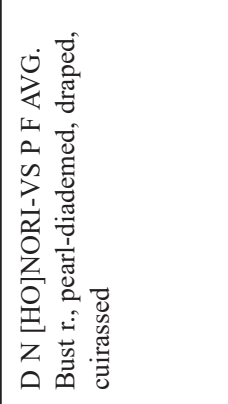 & 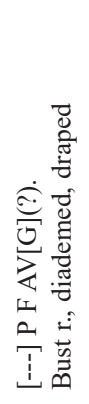 & 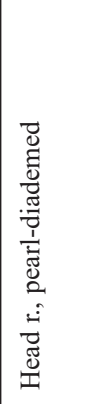 & 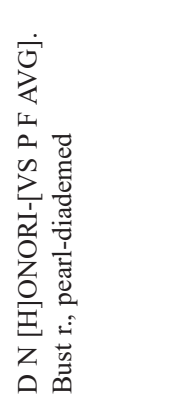 \\
\hline $\begin{array}{l}\dot{g} \\
\dot{\Xi}\end{array}$ & 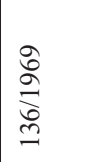 & 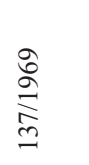 & $\begin{array}{l}\text { वे } \\
\stackrel{\circ}{\circ}\end{array}$ & $\begin{array}{l}\text { वे } \\
\stackrel{2}{a} \\
\text { aे }\end{array}$ & 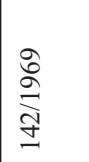 & 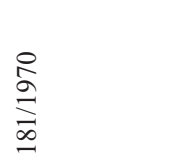 \\
\hline 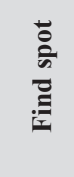 & 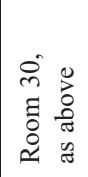 & 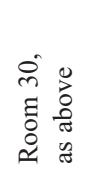 & 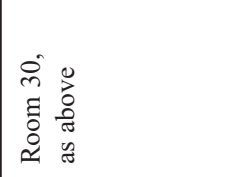 & 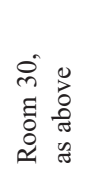 & 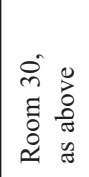 & 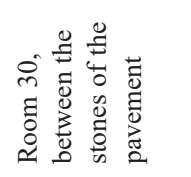 \\
\hline
\end{tabular}




\section{ÉTUDES et TRAVAUX XXXIV / 2021}
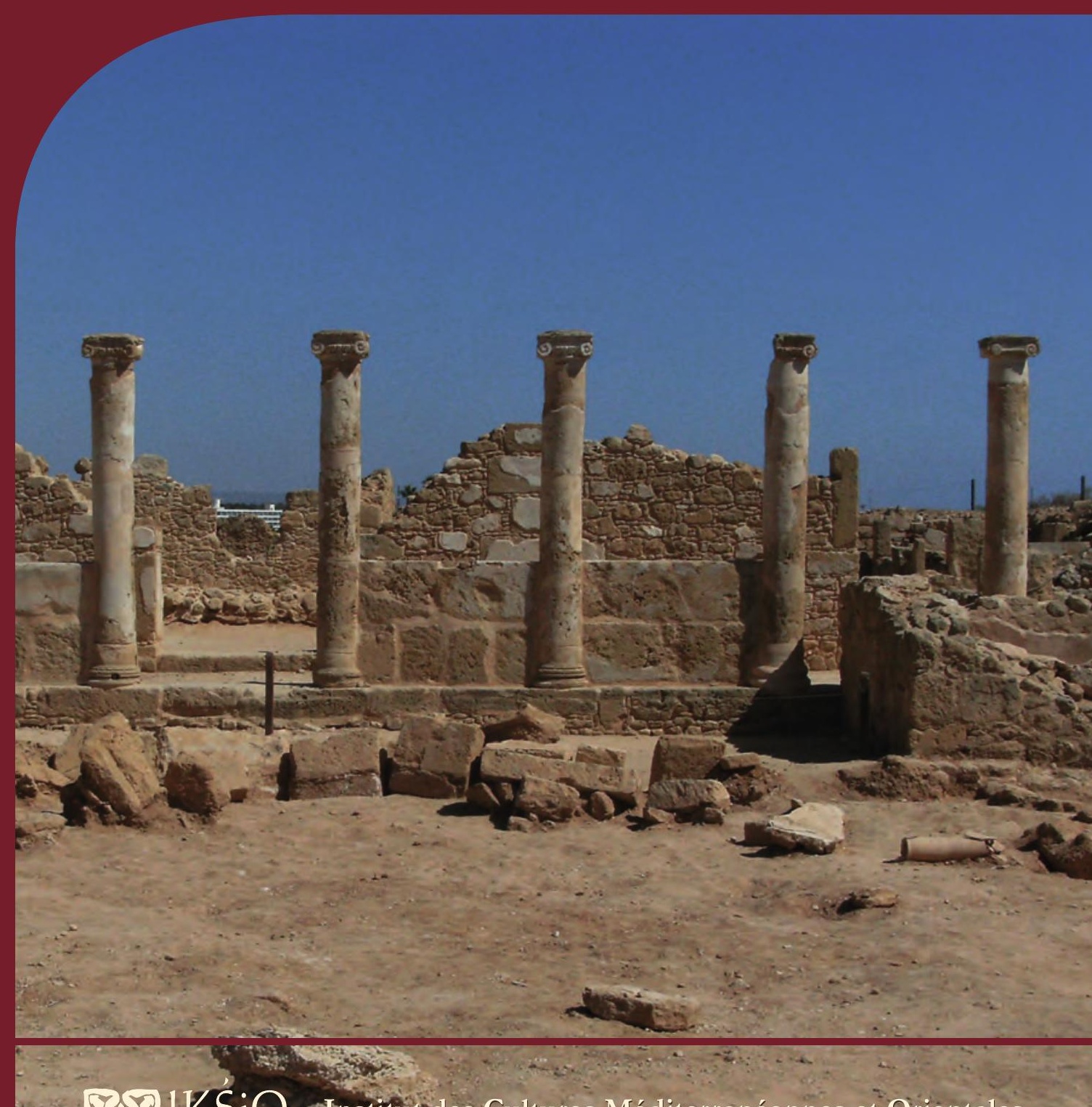

W $92 \mathrm{HKSiO}$ Institut des Cultures Méditerranéennes et Orientales

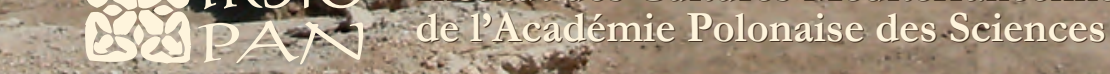

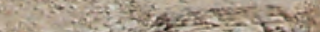

Q 
COMITÉ DE RÉDACTION SCIENTIFIQUE

Maciej Makowski - rédacteur en chef

Jadwiga Iwaszczuk - rédacteur

Katarzyna Kapiec - secrétaire de la rédaction

Henryk Meyza - rédacteur thématique du volume

CONSEIL SCIENTIFIQUE DU JOURNAL

M. Kobusiewicz (IAE PAN, Warszawa)

E. Laskowska-Kusztal (IMOC PAS, Warszawa)

D. Michaelides (University of Cyprus, Nicosia)

J.Ch. Moretti (IRAA-MOM, Université de Lyon 2/CNRS)

D. Raue (Ägyptisches Museum der Universität Leipzig)

P. Reynolds (ICREA, España)

D. Welsby (British Museum, London)

COMITÉ SCIENTIFIQUE DE LECTURE

la liste des membres du comité est accessible en ligne sur

http://www.etudesettravaux.iksiopan.pl

RÉDACTION TECHNIQUE

Marta Kaczanowicz

REVUE DES TEXTES EN ANGLAIS

Jo Harper 
ÉTUDES et TRAVAUX XXXIV 
INSTYTUT KULTUR ŚRÓDZIEMNOMORSKICH I ORIENTALNYCH POLSKIEJ AKADEMII NAUK

\title{
STUDIA i PRACE
}

\section{XXXIV}

\author{
GoIKSiO \\ QSO PAN \\ WARSZAWA \\ 2021
}


INSTITUT DES CULTURES MÉDITERRANÉENNES ET ORIENTALES DE L’ACADÉMIE POLONAISE DES SCIENCES

\section{ÉTUDES et TRAVAUX}

XXXIV

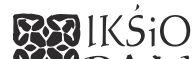

EOSPAN

VARSOVIE

2021 
Publication scientifique financée dans le cadre du programme du Ministre de la Science et de l’Éducation Supérieure

« Programme National de Développement de l’Humanistique » pour les années 2016-2021 (projet no 3bH 150099 83)

\title{
(1) NARODOWY PROGRAM ROZWOJU HUMANISTYKI
}

\author{
Copyright $(C)$ \\ Instytut Kultur Śródziemnomorskich i Orientalnych PAN \\ et les Auteurs \\ Warszawa 2021
}

ISSN 2084-6762

(jusqu'en 2011 : 0079-3566)

e-ISSN 2449-9579
Version première en papier, imprimée en Pologne - 150 copies
Version électronique accessible sur
http://www.etudesettravaux.iksiopan.pl

Édition: Polskie Towarzystwo Historyczne et Wydawnictwo Neriton, Warszawa

Conception générale de la couverture : J. Iwaszczuk

Photographie de couverture : Phot. A. Brzozowska-Jawornicka

(Le portique ionique est, péristyle de la Maison « hellénistique », Nea Paphos) 


\section{Table des matières}

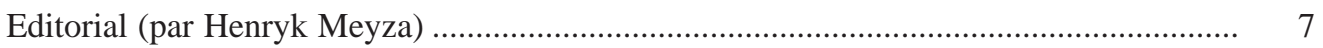

Marta BaJTLER

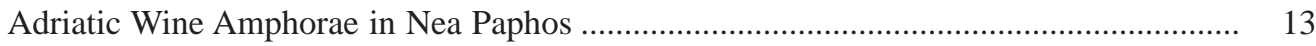

Claire Balandier, Jolanta MlynarczyK

The Temple and Its Surroundings on Fabrika Hill, Paphos: Preliminary Results

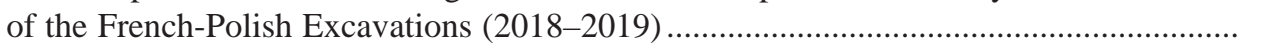

Grażyna BĄKowska-Czerner, Rafal CzERner

The Shell Motif in the Culture and Architecture of the Ancient Town of Marina el-Alamein in Egyp

AleKsandra BrzozowsKa-JaWornicKa

'Hellenistic' House in Nea Paphos, Cyprus - A First Summary of Its Architecture.

AleKsandra BrzozowsKa-JaWORnicKA, AnNa KuBICKA-SowińsKa

In Search of the Module in the Architectural Design of the 'Hellenistic'

House in Nea Paphos, Cyprus

RoKsana HaJduga

Kushite Stamp Impressions from Selib 2, Sudan

ERSIN HusSEIN

Mapping Metal Rich Roman Cyprus: The Case for Object-Centred Approaches

BARBARA LICHOCKA

Villa of Theseus at Nea Paphos (Cyprus). Fourth-Early Fifth Century Numismatic

Evidence for Architectural Transformations and Seismic Events

VASiliki Lysandrou, Demetrios Michaelides

Wall Paintings in Ancient Cyprus: The Hellenistic and Roman Tombs of Paphos

and Its Region

ADAM ŁAJTAR

A Weight of Seleucia in Pieria in Nea Paphos

Diana MroczeK

Ancient Portrait Busts of Marcus Aurelius in the National Museum in Poznań 
Brandon R. Olson, R. Scott Moore, Thomas Landvatter, Justin Stephens

Pyla-Vigla: A Case Study Assessing the Imperial Strategies of the Hellenistic

Diadochoi in Cyprus

Patrizio Pensabene, Eleonora Gasparini

Colonnaded Hall in Kourion: How the Oecus Corinthius Was Interpreted in the Roman

Houses of Cyprus

Monika Rekowska, Demetrios Michaelides, Skevi Christodoulou, JAKUB KANISZEWSKI

Adopting Roman Habits - The Baths in the House of Orpheus in Nea Paphos

as a 'Troublesome' Case Study?

MARCIN M. ROMANIUK

Terracotta Pipelines at Maloutena: Remarks on the Water System in the

Residential District of Ancient Nea Paphos, Cyprus

363

ABRÉVIATIONS

407 